Groups Geom. Dyn. 7 (2013), 293-321

DOI $10.4171 / \mathrm{GGD} / 183$
Groups, Geometry, and Dynamics

(C) European Mathematical Society

\title{
Isometric group actions and the cohomology of flat fiber bundles
}

\author{
Markus Banagl*
}

\begin{abstract}
Using methods originating in the theory of intersection spaces, specifically a de Rham type description of the real cohomology of these spaces by a complex of global differential forms, we show that the Leray-Serre spectral sequence with real coefficients of a flat fiber bundle of smooth manifolds collapses if the fiber is Riemannian and the structure group acts isometrically. The proof is largely topological and does not need a metric on the base or total space. We use this result to show further that if the fundamental group of a smooth aspherical manifold acts isometrically on a Riemannian manifold, then the equivariant real cohomology of the Riemannian manifold can be computed as a direct sum over the cohomology of the group with coefficients in the (generally twisted) cohomology modules of the manifold. Our results have consequences for the Euler class of flat sphere bundles. Several examples are discussed in detail.
\end{abstract}

Mathematics Subject Classification (2010). 55R20, 55R70, 55N91.

Keywords. Serre spectral sequence, cohomology of fiber bundles, flat bundles, isometric group actions, equivariant cohomology, aspherical manifolds, discrete torsion-free transformation groups, Euler class.

\section{Introduction}

A fiber bundle with given structure group is flat if the transition functions into the structure group are locally constant. We show that the method of intersection spaces introduced in [2], specifically the de Rham description of intersection space cohomology given in [3], implies the following result, by a concise and topological proof:

Theorem (Theorem 5.2). Let B, $E$ and $F$ be closed, smooth manifolds with $F$ oriented. Let $\pi: E \rightarrow B$ be a flat, smooth fiber bundle with structure group $H$. If

(1) H is a Lie group acting properly (and smoothly) on F (for example H compact), or

\footnotetext{
* The author was in part supported by a research grant of the Deutsche Forschungsgemeinschaft.
} 
(2) $F$ is Riemannian and the images of the monodromy homomorphisms $\pi_{1}(B, b) \rightarrow$ $H$ act by isometries on $F$, where the base-point $b$ ranges over the connected components of $B$,

then the cohomological Leray-Serre spectral sequence of $\pi$ for real coefficients collapses at the $E_{2}$-term. In particular, the formula

$$
H^{k}(E ; \mathbb{R}) \cong \bigoplus_{p+q=k} H^{p}\left(B ; \boldsymbol{H}^{q}(F ; \mathbb{R})\right)
$$

holds, where the $\boldsymbol{H}^{q}(F ; \mathbb{R})$ are local coefficient systems on $B$ induced by $\pi$, whose groups are the real cohomology groups of the fiber.

By a result of R. Palais ([15]), condition (1) implies that $F$ can be endowed with a Riemannian metric such that (2) holds. The isometry group of an $m$-dimensional, compact, Riemannian manifold is a compact Lie group of dimension at most $\frac{1}{2} m(m+1)$. Thus (2) implies (1) by taking $H$ to be the isometry group of $F$. In Section 6, we provide an example of a flat, smooth fiber bundle whose structure group does not act isometrically for any Riemann metric on the fiber, and whose spectral sequence does not collapse at $E_{2}$. Hence, conditions (1), (2) in the theorem cannot be deleted without substitution.

Let us indicate two immediate consequences of the theorem. Let $\pi: E \rightarrow B$ be an oriented, flat sphere bundle with structure group $\mathrm{SO}(n)$ over a closed, smooth manifold $B$. The transgression

$$
\begin{gathered}
d_{n}: E_{n}^{0, n-1}=H^{0}\left(B ; \boldsymbol{H}^{n-1}\left(S^{n-1} ; \mathbb{R}\right)\right)=H^{n-1}\left(S^{n-1} ; \mathbb{R}\right) \\
\longrightarrow E_{n}^{n, 0}=H^{n}\left(B ; \boldsymbol{H}^{0}\left(S^{n-1} ; \mathbb{R}\right)\right)=H^{n}(B ; \mathbb{R})
\end{gathered}
$$

sends a certain element in $E_{1}^{0, n-1}$, which corresponds to local angular forms on the sphere bundle, and which survives to $E_{n}$, to the Euler class of the sphere bundle. Since $\mathrm{SO}(n)$ is compact, the spectral sequence of the bundle collapses at $E_{2}$, by the theorem. Thus $d_{n}=0$ and we obtain the following corollary.

Corollary. The real Euler class of an oriented, flat, linear sphere bundle (structure group $\mathrm{SO}(n)$ ) over a closed, smooth manifold is zero.

We thus obtain a topological proof, without using the Chern-Weil theory of curvature forms, of a result closely related to the results of [12], Section 4, which do rely on Chern-Weil theory.

By [16], there is a manifold $M^{2 n}$ with flat tangent bundle and nonzero Euler characteristic for every $n>1$. If the associated tangent sphere bundle of $M$, with structure group $\mathrm{SO}(2 n)$, were flat, then the Euler class of such a sphere bundle would vanish according to the above corollary. We arrive at: 
Corollary. For every $n>1$, there is a manifold $M^{2 n}$ with flat tangent bundle, whose linear tangent sphere bundle is not flat.

For the tangent bundles of these manifolds $M^{2 n}$, the structure group $\mathrm{GL}^{+}(2 n, \mathbb{R})$ can be reduced to $\mathrm{GL}^{+}(2 n, \mathbb{R})$ with the discrete topology and can also be reduced to $\mathrm{SO}(2 n)$ (by using a metric), but there are no further reductions to $\mathrm{SO}(2 n)$ with the discrete topology.

Our theorem may be applied to the equivariant cohomology $H_{G}^{\bullet}(-; \mathbb{R})$ of certain discrete groups $G$ :

Theorem (Theorem 7.1). Let $F$ be an oriented, closed, smooth manifold and $G$ a discrete group, whose Eilenberg-MacLane space $K(G, 1)$ may be taken to be a closed, smooth manifold. If for a smooth action of $G$ on $F$,

(1) the action factors through a proper, smooth Lie group action, or

(2) $F$ is Riemannian and $G$ acts isometrically on $F$,

then there is a decomposition

$$
H_{G}^{k}(F ; \mathbb{R}) \cong \bigoplus_{p+q=k} H^{p}\left(G ; \boldsymbol{H}^{q}(F ; \mathbb{R})\right),
$$

where the $\boldsymbol{H}^{q}(F ; \mathbb{R})$ are $G$-modules determined by the action.

The assumption on $G$ can be paraphrased as requiring $G$ to be the fundamental group of a closed, (smooth) aspherical manifold. Examples of such groups include finitely generated free abelian groups, the fundamental groups of closed manifolds with non-positive sectional curvature, the fundamental groups of surfaces other than the real projective plane, infinite fundamental groups of irreducible, closed, orientable 3-manifolds, torsion-free discrete subgroups of almost connected Lie groups, and certain groups arising from Gromov's hyperbolization technique. Only torsion-free $G$ can satisfy the hypothesis of the above theorem. Note that in (1) we do not assume that the image of $G$ is closed in the intermediary Lie group, nor that one can identify $G$ with a subgroup of the intermediary group, and in (2) we do not assume that the image of $G$ is closed in the isometry group of $F$, nor that one can identify $G$ with a subgroup of the isometries. For instance, the integers $G=\mathbb{Z}$ with $K(\mathbb{Z}, 1)=S^{1}$, the circle, act isometrically and freely (and ergodically) on the unit circle by powers of a rotation by an angle which is an irrational multiple of $2 \pi$. Theorem 7.1 thus emphasizes the discrete dynamical systems viewpoint. This example also satisfies (1), since the powers of the irrational rotation are a subgroup (which is not closed) of the compact Lie group $S^{1}$ which acts on itself by (e.g. left) multiplication. The actions to which the theorem applies need not be proper, nor need our $G$-spaces be $G$-CW complexes, but in many geometric situations, nonproper actions factor through proper actions in a natural way. (The above $\mathbb{Z}$-action on the circle is not proper, since e.g. the orbit space is not Hausdorff and orbits are not closed in $S^{1}$.) Section 7 contains 
a number of corollaries to, applications of, and examples illustrating Theorem 7.1. For instance, if an integral Heisenberg group $\mathfrak{S}_{n}$ acts isometrically on an oriented, closed, connected, Riemannian manifold $F$, then

$$
\text { rk } H_{\mathfrak{S}_{n}}^{k}(F) \geq 2, \quad k=1,2,
$$

and $H_{\mathfrak{S}_{n}}^{3}(F ; \mathbb{R})$ does not vanish (Corollary 7.8).

In Section 8, we illustrate the use of our results by calculating explicitly an equivariant cohomology group of a certain action of a free abelian group on the 4-manifold $F=S^{2} \times S^{2}$. The action in this example does not factor through a finite group.

The proof of Theorem 5.2 relies on the complex of multiplicatively structured forms constructed in [3], and the fact that fiberwise truncation in both directions, yielding a subcomplex in both cases (and not a quotient complex in one case), can be performed on the multiplicatively structured forms. Such truncations, carried out homotopy theoretically on the space level (generally a much harder problem), are also required to build the intersection space $I^{\bar{p}} X$ of a stratified pseudomanifold $X$. The only analytic tool we need to prove the theorem is the classical Hodge decomposition - hence the assumption that the fiber must be closed, oriented and Riemannian. Otherwise, our argument is purely topological in nature, using Čech complexes. No connections, tensor fields, etc. are used on the base or total space; in particular we need not assume that $B$ and $E$ are Riemannian. The combinatorial nature of our proof may lend itself to an extension of our theorem to non-smoothable PL manifolds $B$ and $E$ and flat PL bundles $\pi: E \rightarrow B$. In this situation, smooth forms have to be replaced by Sullivan's complex $\tilde{A}^{\bullet}(K)$ of piecewise $C^{\infty}$-forms on a simplicial complex $K$. We may also recall at this point that there are closed, aspherical PL manifolds which are not homotopy equivalent to closed, smooth manifolds, [7].

Our Theorem 5.2 is closely related to results of [6] and [14]. Dai and Müller work with Riemannian $E, B$ and a Riemannian submersion $\pi: E \rightarrow B$. Using Dai's spectral sequence degeneration result from [6], Müller proves that if a flat Riemannian submersion $\pi$ has totally geodesic fibers, then the spectral sequence of $\pi$ collapses at $E_{2}$. Let us put this into perspective. A Riemannian submersion whose total space is complete is a locally trivial fiber bundle. The geometry of a Riemannian submersion is largely governed by two tensor fields $T$ and $A$. Let $\mathcal{V}$ and $\mathscr{H}$ denote the vertical and horizontal distributions, respectively, that is, at each point $x \in E$ there is an orthogonal decomposition $\mathcal{V}_{x} \oplus \mathscr{H}_{x}=T_{x} E$ of the tangent space and $\mathcal{V}_{x}$ is tangent to the fiber over $\pi(x)$. Let $\mathcal{V}$ and $\mathscr{H}$ also denote the projection of a vector onto $\mathcal{V}_{x}$ and $\mathscr{H}_{x}$, respectively. With $\nabla$ the Levi-Civita connection of the metric on $E$, one sets for vector fields $V, W$ on $E$,

$$
T_{V} W=\mathscr{H} \nabla_{\mathcal{V} V} \mathcal{V} W+\mathcal{V} \nabla_{\mathcal{V} V} \mathscr{H} W
$$

and

$$
A_{V} W=\mathscr{H} \nabla_{\mathscr{H} V} \mathcal{V} W+\mathcal{V} \nabla_{\mathscr{H} V} \mathscr{H} W .
$$


If $V, W$ are vertical, then $T_{V} W$ is the second fundamental form of each fiber. The identical vanishing $T=0$ is equivalent to each fiber being totally geodesic, that is, geodesics in the fibers are also geodesics for $E$. This implies that all fibers are isometric and the holonomy group (which agrees with the structure group of $\pi$, at least when $E$ is complete) is a subgroup of the isometry group of the fiber. The identical vanishing $A=0$ is equivalent to the integrability of $\mathscr{H}$. If $\mathscr{H}$ is integrable, then $E$ is locally isometric to $B \times F$ with a metric $g_{B}+g_{F, b}$, where $g_{B}$ is a metric on $B$ and $\left\{g_{F, b}\right\}_{b \in B}$ is a smooth family of metrics on $F$. In this situation, the horizontal foliation yields a flat (Ehresmann) connection for $\pi$. For a flat connection, the holonomy along a path depends only on the homotopy class of the path. Indeed, for path-connected $B$, flat bundles with structure group $G$ acting effectively on a fiber $F$ are in one-to-one correspondence with homomorphisms $\pi_{1}(B) \rightarrow G$. In particular, one may take $G$ to be discrete. A warped product is a Riemannian manifold $B \times F$, whose metric has the form $g_{B}+f g_{F}$, where $g_{F}$ is a fixed metric on $F$ and $f: B \rightarrow \mathbb{R}$ is a positive function. If the Riemannian submersion $\pi$ is locally a warped product, then $\mathscr{H}$ is integrable. When $T \equiv 0$ and $A \equiv 0$, the total space $E$ is locally a product $g_{B}+g_{F}$, where $g_{F}$ does not depend on points in the base. From [6], Müller isolates a technical condition, called condition "(B)" in [14], Section 2.3, which for a flat Riemannian submersion implies collapse of the spectral sequence at $E_{2}$. He shows that this condition is satisfied for warped products as well as for totally geodesic fibers, while Dai shows that collapse at $E_{2}$ happens for flat Riemannian submersions satisfying (B).

\section{A complex of multiplicatively structured forms on flat bundles}

This section reviews the multiplicatively structured form model introduced in [3]. The proofs of the cited results can be found in that paper. For a smooth manifold $M, \Omega^{\bullet}(M)$ denotes the de Rham complex of smooth differential forms on $M$. Let $F$ be a closed, oriented, Riemannian manifold and $\pi: E \rightarrow B$ a flat, smooth fiber bundle over the closed, smooth $n$-dimensional base manifold $B$ with fiber $F$ and structure group the isometries of $F$. An open cover of an $n$-manifold is called good if all nonempty finite intersections of sets in the cover are diffeomorphic to $\mathbb{R}^{n}$. Every smooth manifold has a good cover and if the manifold is compact, then the cover can be chosen to be finite. Let $\mathcal{U}=\left\{U_{\alpha}\right\}$ be a finite good open cover of the base $B$ such that $\pi$ trivializes with respect to $\mathcal{U}$. Let $\left\{\phi_{\alpha}\right\}$ be a system of local trivializations, that is, the $\phi_{\alpha}$ are diffeomorphisms such that

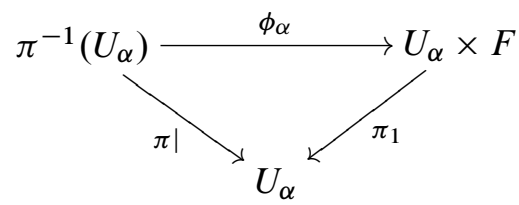


commutes for every $\alpha$. Flatness implies that the transition functions

$$
\rho_{\beta \alpha}=\phi_{\beta}\left|\circ \phi_{\alpha}\right|^{-1}:\left(U_{\alpha} \cap U_{\beta}\right) \times F \rightarrow \pi^{-1}\left(U_{\alpha} \cap U_{\beta}\right) \rightarrow\left(U_{\alpha} \cap U_{\beta}\right) \times F
$$

are of the form

$$
\rho_{\beta \alpha}(t, x)=\left(t, g_{\beta \alpha}(x)\right) .
$$

The maps $g_{\beta \alpha}: F \rightarrow F$ are isometries.

If $X$ is a topological space, let $\pi_{2}: X \times F \rightarrow F$ denote the second-factor projection. Let $V \subset B$ be a $\mathfrak{U}$-small open subset and suppose that $V \subset U_{\alpha}$.

Definition 2.1. A differential form $\omega \in \Omega^{q}\left(\pi^{-1}(V)\right)$ is called $\alpha$-multiplicatively structured if it has the form

$$
\omega=\phi_{\alpha}^{*} \sum_{j} \pi_{1}^{*} \eta_{j} \wedge \pi_{2}^{*} \gamma_{j}, \quad \eta_{j} \in \Omega^{\bullet}(V), \gamma_{j} \in \Omega^{\bullet}(F)
$$

(finite sums).

Flatness is crucial for the following basic lemma.

Lemma 2.2. Suppose that $V \subset U_{\alpha} \cap U_{\beta}$. Then $\omega$ is $\alpha$-multiplicatively structured if, and only if, $\omega$ is $\beta$-multiplicatively structured.

The lemma follows from the transformation law

$$
\phi_{\alpha}^{*} \sum_{j} \pi_{1}^{*} \eta_{j} \wedge \pi_{2}^{*} \gamma_{j}=\phi_{\beta}^{*} \sum_{j} \pi_{1}^{*} \eta_{j} \wedge \pi_{2}^{*}\left(g_{\alpha \beta}^{*} \gamma_{j}\right)
$$

The lemma shows that the property of being multiplicatively structured over $V$ is invariantly defined, independent of the choice of $\alpha$ such that $V \subset U_{\alpha}$. We will use the shorthand notation

$$
U_{\alpha_{0} \ldots \alpha_{p}}=U_{\alpha_{0}} \cap \cdots \cap U_{\alpha_{p}}
$$

for multiple intersections. (Repetitions of indices are allowed.) Since $\mathfrak{U}$ is a good cover, every $U_{\alpha_{0} \ldots \alpha_{p}}$ is diffeomorphic to $\mathbb{R}^{n}, n=\operatorname{dim} B$. A linear subspace, the subspace of multiplicatively structured forms, of $\Omega^{q}(E)$ is obtained by setting

$$
\Omega_{\mathcal{M S}}^{q}(B)=\left\{\omega \in \Omega^{q}(E)|\omega|_{\pi^{-1} U_{\alpha}} \text { is } \alpha \text {-multiplicatively structured for all } \alpha\right\} .
$$

The exterior derivative $d: \Omega^{q}(E) \rightarrow \Omega^{q+1}(E)$ restricts to a differential

$$
d: \Omega_{\mathcal{M S}}^{q}(B) \rightarrow \Omega_{\mathcal{M S}}^{q+1}(B) .
$$

Thus $\Omega_{\mathcal{M S}}^{\bullet}(B) \subset \Omega^{\bullet}(E)$ is a subcomplex. We shall eventually see that this inclusion is a quasi-isomorphism, that is, induces isomorphisms on cohomology. For any $\alpha$, set

$$
\Omega_{\mathcal{M S}}^{\bullet}\left(U_{\alpha}\right)=\left\{\omega \in \Omega^{\bullet}\left(\pi^{-1} U_{\alpha}\right) \mid \omega \text { is } \alpha \text {-multiplicatively structured }\right\}
$$


Let $r$ denote the obvious restriction map

$$
r: \Omega_{\mathcal{M} S}^{\bullet}(B) \rightarrow \prod_{\alpha} \Omega_{\mathcal{M} S}^{\bullet}\left(U_{\alpha}\right) .
$$

If $p$ is positive, then we set

$$
\Omega_{\mathcal{M S}}^{\bullet}\left(U_{\alpha_{0} \ldots \alpha_{p}}\right)=\left\{\omega \in \Omega^{\bullet}\left(\pi^{-1} U_{\alpha_{0} \ldots \alpha_{p}}\right) \mid \omega \text { is } \alpha_{0} \text {-multiplicatively structured }\right\} .
$$

Lemma 2.2 implies that for any $1 \leq j \leq p$,

$$
\Omega_{\mathcal{M S}}^{\bullet}\left(U_{\alpha_{0} \ldots \alpha_{p}}\right)=\left\{\omega \in \Omega^{\bullet}\left(\pi^{-1} U_{\alpha_{0} \ldots \alpha_{p}}\right) \mid \omega \text { is } \alpha_{j} \text {-multiplicatively structured }\right\} .
$$

In particular, if $\sigma$ is any permutation of $0,1, \ldots, p$, then

$$
\Omega_{\mathcal{M S}}^{\bullet}\left(U_{\alpha_{\sigma(0)} \ldots \alpha_{\sigma(p)}}\right)=\Omega_{\mathcal{M S}}^{\bullet}\left(U_{\alpha_{0} \ldots \alpha_{p}}\right) .
$$

The components of an element

$$
\xi \in \prod_{\alpha_{0}, \ldots, \alpha_{p}} \Omega_{\mathcal{M S}}^{\bullet}\left(U_{\alpha_{0} \ldots \alpha_{p}}\right)
$$

will be written as

$$
\xi_{\alpha_{0} \ldots \alpha_{p}} \in \Omega_{\mathcal{M} \mathcal{\bullet}}^{\bullet}\left(U_{\alpha_{0} \ldots \alpha_{p}}\right) .
$$

We impose the antisymmetry restriction $\xi_{\ldots \alpha_{i} \ldots \alpha_{j} \ldots}=-\xi_{\ldots \alpha_{j} \ldots \alpha_{i} \ldots}$ upon interchange of two indices. In particular, if $\alpha_{0}, \ldots, \alpha_{k}$ contains a repetition, then $\xi_{\alpha_{0} \ldots \alpha_{k}}=0$. The difference operator

$$
\delta: \prod \Omega^{\bullet}\left(\pi^{-1} U_{\alpha_{0} \ldots \alpha_{p}}\right) \rightarrow \prod \Omega^{\bullet}\left(\pi^{-1} U_{\alpha_{0} \ldots \alpha_{p+1}}\right),
$$

defined by

$$
(\delta \xi)_{\alpha_{0} \ldots \alpha_{p+1}}=\left.\sum_{j=0}^{p+1}(-1)^{j} \xi_{\alpha_{0} \ldots \hat{\alpha}_{j} \ldots \alpha_{p+1}}\right|_{\pi^{-1} U_{\alpha_{0} \ldots \alpha_{p+1}}}
$$

and satisfying $\delta^{2}=0$, restricts to a difference operator

$$
\delta: \prod \Omega_{\mathcal{M} S}^{\bullet}\left(U_{\alpha_{0} \ldots \alpha_{p}}\right) \rightarrow \prod \Omega_{\mathcal{M} S}^{\bullet}\left(U_{\alpha_{0} \ldots \alpha_{p+1}}\right) .
$$

Since the de Rham differential $d$ commutes with restriction to open subsets, we have $d \delta=\delta d$. Thus

$$
C^{p}\left(\mathcal{U} ; \Omega_{\mathcal{M S}}^{q}\right)=\prod \Omega_{\mathcal{M} S}^{q}\left(U_{\alpha_{0} \ldots \alpha_{p}}\right)
$$

is a double complex with horizontal differential $\delta$ and vertical differential $d$. The associated simple complex $C_{\mathcal{M} S}^{\bullet}(\mathfrak{U})$ has groups

$$
C_{\mathcal{M S}}^{j}(\mathcal{U})=\bigoplus_{p+q=j} C^{p}\left(\mathfrak{U} ; \Omega_{\mathcal{M S}}^{q}\right)
$$


in degree $j$ and differential $D=\delta+(-1)^{p} d$ on $C^{p}\left(\mathfrak{U} ; \Omega_{\mathcal{M S}}^{q}\right)$. We shall refer to the double complex $\left(C^{\bullet}\left(\mathfrak{U} ; \Omega_{\mathcal{M S}}^{\bullet}\right), \delta, d\right)$ as the multiplicatively structured $\check{C} e c h-d e$ Rham complex.

Lemma 2.3 (Generalized Mayer-Vietoris sequence). The sequence

$$
0 \rightarrow \Omega_{\mathcal{M S}}^{\bullet}(B) \stackrel{r}{\rightarrow} C^{0}\left(\mathfrak{U} ; \Omega_{\mathcal{M S}}^{\bullet}\right) \stackrel{\delta}{\rightarrow} C^{1}\left(\mathfrak{U} ; \Omega_{\mathcal{M S}}^{\bullet} \stackrel{\stackrel{\delta}{\rightarrow}}{\rightarrow} C^{2}\left(\mathfrak{U} ; \Omega_{\mathcal{M S}}^{\bullet}\right) \stackrel{\delta}{\rightarrow} \cdots\right.
$$

is exact.

Let us recall a fundamental fact about double complexes.

Proposition 2.4. If all the rows of an augmented double complex are exact, then the augmentation map induces an isomorphism from the cohomology of the augmentation column to the cohomology of the simple complex associated to the double complex.

This fact is applied in showing:

Proposition 2.5. The restriction map $r: \Omega_{\mathcal{M S}}^{\bullet}(B) \rightarrow C^{0}\left(\mathfrak{U} ; \Omega_{\mathcal{M S}}^{\bullet}\right)$ induces an isomorphism

$$
r^{*}: H^{\bullet}\left(\Omega_{\mathcal{M S}}^{\bullet}(B)\right) \stackrel{\cong}{\Longrightarrow} H^{\bullet}\left(C_{\mathcal{M S}}^{\bullet}(\mathfrak{U}), D\right) .
$$

The double complex $\left(C^{\bullet}\left(\pi^{-1} \mathfrak{U} ; \Omega^{\bullet}\right), \delta, d\right)$ given by

$$
C^{p}\left(\pi^{-1} \mathfrak{U} ; \Omega^{q}\right)=\prod \Omega^{q}\left(\pi^{-1} U_{\alpha_{0} \ldots \alpha_{p}}\right)
$$

can be used to compute the cohomology of the total space $E$. The restriction map

$$
\bar{r}: \Omega^{\bullet}(E) \rightarrow \prod_{\alpha} \Omega^{\bullet}\left(\pi^{-1} U_{\alpha}\right)=C^{0}\left(\pi^{-1} \mathfrak{U} ; \Omega^{\bullet}\right)
$$

makes $C^{\bullet}\left(\pi^{-1} \mathfrak{U} ; \Omega^{\bullet}\right)$ into an augmented double complex. By the generalized MayerVietoris sequence, Proposition 8.5 of [4], the rows of this augmented double complex are exact. From Proposition 2.4, we thus deduce:

Proposition 2.6. The restriction map $\bar{r}: \Omega^{\bullet}(E) \rightarrow C^{0}\left(\pi^{-1} \mathfrak{U} ; \Omega^{\bullet}\right)$ induces an isomorphism

$$
\bar{r}^{*}: H^{\bullet}(E)=H^{\bullet}\left(\Omega^{\bullet}(E)\right) \stackrel{\cong}{\cong} H^{\bullet}\left(C^{\bullet}\left(\pi^{-1} \mathfrak{U}\right), D\right),
$$

where $\left(C^{\bullet}\left(\pi^{-1} \mathfrak{U}\right), D\right)$ is the simple complex of $\left(C^{\bullet}\left(\pi^{-1} \mathfrak{U} ; \Omega^{\bullet}\right), \delta, d\right)$.

Regarding $\mathbb{R}^{n} \times F$ as a trivial fiber bundle over $\mathbb{R}^{n}$ with projection $\pi_{1}$, the multiplicatively structured complex $\Omega_{\mathcal{M S}}^{\bullet}\left(\mathbb{R}^{n}\right)$ is defined by

$\Omega_{\mathcal{M} \mathcal{S}}^{\bullet}\left(\mathbb{R}^{n}\right)=\left\{\omega \in \Omega^{\bullet}\left(\mathbb{R}^{n} \times F\right) \mid \omega=\sum_{j} \pi_{1}^{*} \eta_{j} \wedge \pi_{2}^{*} \gamma_{j}, \eta_{j} \in \Omega^{\bullet}\left(\mathbb{R}^{n}\right), \gamma_{j} \in \Omega^{\bullet}(F)\right\}$. 
Let $s: \mathbb{R}^{n-1} \hookrightarrow \mathbb{R} \times \mathbb{R}^{n-1}=\mathbb{R}^{n}$ be the standard inclusion $s(u)=(0, u), u \in \mathbb{R}^{n-1}$. Let $q: \mathbb{R}^{n}=\mathbb{R} \times \mathbb{R}^{n-1} \rightarrow \mathbb{R}^{n-1}$ be the standard projection $q(t, u)=u$, so that $q s=\mathrm{id}_{\mathbb{R}^{n-1}}$. Set

$$
S=s \times \operatorname{id}_{F}: \mathbb{R}^{n-1} \times F \hookrightarrow \mathbb{R}^{n} \times F, \quad Q=q \times \operatorname{id}_{F}: \mathbb{R}^{n} \times F \rightarrow \mathbb{R}^{n-1} \times F
$$

so that $Q S=\operatorname{id}_{\mathbb{R}^{n-1} \times F}$. The induced map $S^{*}: \Omega^{\bullet}\left(\mathbb{R}^{n} \times F\right) \rightarrow \Omega^{\bullet}\left(\mathbb{R}^{n-1} \times F\right)$ restricts to a map

$$
S^{*}: \Omega_{\mathcal{M S}}^{\bullet}\left(\mathbb{R}^{n}\right) \rightarrow \Omega_{\mathcal{M S}}^{\bullet}\left(\mathbb{R}^{n-1}\right) .
$$

The induced map $Q^{*}: \Omega^{\bullet}\left(\mathbb{R}^{n-1} \times F\right) \rightarrow \Omega^{\bullet}\left(\mathbb{R}^{n} \times F\right)$ restricts to a map

$$
Q^{*}: \Omega_{\mathcal{M S}}^{\bullet}\left(\mathbb{R}^{n-1}\right) \rightarrow \Omega_{\mathcal{M S}}^{\bullet}\left(\mathbb{R}^{n}\right) .
$$

Proposition 2.7. The maps

$$
\Omega_{\mathcal{M S}}^{\bullet}\left(\mathbb{R}^{n}\right) \stackrel{S^{*}}{\underset{Q^{*}}{\leftrightarrows}} \Omega_{\mathcal{M S}}^{\bullet}\left(\mathbb{R}^{n-1}\right)
$$

are chain homotopy inverses of each other and thus induce mutually inverse isomorphisms

$$
H^{\bullet}\left(\Omega_{\mathcal{M S}}^{\bullet}\left(\mathbb{R}^{n}\right)\right) \underset{Q^{*}}{\stackrel{S^{*}}{\rightleftarrows}} H^{\bullet}\left(\Omega_{\mathcal{M S}}^{\bullet}\left(\mathbb{R}^{n-1}\right)\right)
$$

on cohomology.

Let $S_{0}: F=\{0\} \times F \hookrightarrow \mathbb{R}^{n} \times F$ be the inclusion at 0 , inducing a map $S_{0}^{*}: \Omega_{\mathcal{M S}}^{*}\left(\mathbb{R}^{n}\right) \rightarrow \Omega^{\bullet}(F)$. The map $\pi_{2}^{*}: \Omega^{\bullet}(F) \rightarrow \Omega^{\bullet}\left(\mathbb{R}^{n} \times F\right)$ restricts to a map $\pi_{2}^{*}: \Omega^{\bullet}(F) \rightarrow \Omega_{\mathcal{M S}}^{*}\left(\mathbb{R}^{n}\right)$. An induction on $n$ using Proposition 2.7 shows:

Proposition 2.8 (Poincaré lemma for multiplicatively structured forms). The maps

$$
\Omega_{\mathcal{M S}}^{\bullet}\left(\mathbb{R}^{n}\right) \underset{\pi_{2}^{*}}{\stackrel{S_{0}^{*}}{\rightleftarrows}} \Omega^{\bullet}(F)
$$

are chain homotopy inverses of each other and thus induce mutually inverse isomorphisms

$$
H^{\bullet}\left(\Omega_{\mathcal{M S}}^{\bullet}\left(\mathbb{R}^{n}\right)\right) \underset{\pi_{2}^{*}}{\stackrel{S_{0}^{*}}{\rightleftarrows}} H^{\bullet}(F)
$$

on cohomology.

Using the classical Poincaré lemma, this proposition readily implies: 
Proposition 2.9. The inclusion $\Omega_{\mathcal{M} S}^{\bullet}\left(\mathbb{R}^{n}\right) \subset \Omega^{\bullet}\left(\mathbb{R}^{n} \times F\right)$ induces an isomorphism

$$
H^{\bullet}\left(\Omega_{\mathcal{M} S}^{\bullet}\left(\mathbb{R}^{n}\right)\right) \cong H^{\bullet}\left(\mathbb{R}^{n} \times F\right)
$$

on cohomology.

Proposition 2.10. For any $U_{\alpha_{0} \ldots \alpha_{p}}$, the inclusion

$$
\Omega_{\mathcal{M} S}^{\bullet}\left(U_{\alpha_{0} \ldots \alpha_{p}}\right) \hookrightarrow \Omega^{\bullet}\left(\pi^{-1} U_{\alpha_{0} \ldots \alpha_{p}}\right)
$$

induces an isomorphism on cohomology (with respect to the de Rham differential $d$ ).

Since $d$ and $\delta$ on the double complex $C^{\bullet}\left(\mathcal{U} ; \Omega_{\mathcal{M S}}^{\bullet}\right)$ were obtained by restricting $d$ and $\delta$ on $C^{\bullet}\left(\pi^{-1} \mathfrak{U} ; \Omega^{\bullet}\right)$, the natural inclusion $C^{\bullet}\left(\mathfrak{U} ; \Omega_{\mathcal{M S}}^{\bullet}\right) \hookrightarrow C^{\bullet}\left(\pi^{-1} \mathfrak{U} ; \Omega^{\bullet}\right)$ is a morphism of double complexes. It induces an isomorphism on vertical (that is, $d$-)cohomology by Proposition 2.10. Whenever a morphism of double complexes induces an isomorphism on vertical cohomology, then it also induces an isomorphism on the $D$-cohomology of the respective simple complexes. Consequently, using Propositions 2.5 and 2.6, one gets:

Theorem 2.11. The inclusion $\Omega_{\mathcal{M S}}^{\bullet}(B) \hookrightarrow \Omega^{\bullet}(E)$ induces an isomorphism

$$
H^{\bullet}\left(\Omega_{\mathcal{M S}}^{\bullet}(B)\right) \stackrel{\cong}{\longrightarrow} H^{\bullet}(E)
$$

on cohomology.

\section{Fiberwise truncation}

Before we discuss fiberwise (co)truncation, we must first discuss (co)truncation over a point. Again, we refer to [3] for complete proofs of the facts cited in this section. We shall use the Riemannian metric on $F$ to define truncation $\tau_{<k}$ and cotruncation $\tau_{\geq k}$ of the complex $\Omega^{\bullet}(F)$. The bilinear form

$$
(\cdot, \cdot): \Omega^{r}(F) \times \Omega^{r}(F) \rightarrow \mathbb{R}, \quad(\omega, \eta) \mapsto \int_{F} \omega \wedge * \eta,
$$

where $*$ is the Hodge star, is symmetric and positive definite, thus defines an inner product on $\Omega^{\bullet}(F)$. The codifferential

$$
d^{*}=(-1)^{m(r+1)+1} * d *: \Omega^{r}(F) \rightarrow \Omega^{r-1}(F)
$$

is the adjoint of the differential $d,(d \omega, \eta)=\left(\omega, d^{*} \eta\right)$. The classical Hodge decomposition theorem provides orthogonal splittings

$$
\begin{aligned}
\Omega^{r}(F) & =\operatorname{im} d^{*} \oplus \operatorname{Harm}^{r}(F) \oplus \operatorname{im} d, \\
\operatorname{ker} d & =\operatorname{Harm}^{r}(F) \oplus \operatorname{im} d, \\
\operatorname{ker} d^{*} & =\operatorname{im} d^{*} \oplus \operatorname{Harm}^{r}(F),
\end{aligned}
$$


where $\operatorname{Harm}^{r}(F)=\operatorname{ker} d \cap \operatorname{ker} d^{*}$ are the closed and coclosed, i.e. harmonic, forms on $F$. In particular,

$$
\Omega^{r}(F)=\operatorname{im} d^{*} \oplus \operatorname{ker} d=\operatorname{ker} d^{*} \oplus \operatorname{im} d .
$$

Let $k$ be a non-negative integer.

Definition 3.1. The truncation $\tau_{<k} \Omega^{\bullet}(F)$ of $\Omega^{\bullet}(F)$ is the complex

$$
\tau_{<k} \Omega^{\bullet}(F)=\cdots \rightarrow \Omega^{k-2}(F) \rightarrow \Omega^{k-1}(F) \stackrel{d^{k-1}}{\longrightarrow} \operatorname{im} d^{k-1} \rightarrow 0 \rightarrow 0 \rightarrow \cdots,
$$

where $\operatorname{im} d^{k-1} \subset \Omega^{k}(F)$ is placed in degree $k$.

The inclusion $\tau_{<k} \Omega^{\bullet}(F) \subset \Omega^{\bullet}(F)$ is a morphism of complexes. The induced map on cohomology, $H^{r}\left(\tau_{<k} \Omega^{\bullet} F\right) \rightarrow H^{r}(F)$, is an isomorphism for $r<k$, while $H^{r}\left(\tau_{<k} \Omega^{\bullet} F\right)=0$ for $r \geq k$.

Definition 3.2. The cotruncation $\tau_{\geq k} \Omega^{\bullet}(F)$ of $\Omega^{\bullet}(F)$ is the complex

$$
\tau_{\geq k} \Omega^{\bullet}(F)=\cdots \rightarrow 0 \rightarrow 0 \rightarrow \operatorname{ker} d^{*} \stackrel{d^{k} \mid}{\longrightarrow} \Omega^{k+1}(F) \stackrel{d^{k+1}}{\longrightarrow} \Omega^{k+2}(F) \rightarrow \cdots,
$$

where ker $d^{*} \subset \Omega^{k}(F)$ is placed in degree $k$.

The inclusion $\tau_{\geq k} \Omega^{\bullet}(F) \subset \Omega^{\bullet}(F)$ is a morphism of complexes. By construction, we have $H^{r}\left(\tau_{\geq k} \Omega^{\circ} F\right)=0$ for $r<k$ and the inclusion $\tau_{\geq k} \Omega^{\bullet}(F) \hookrightarrow \Omega^{\bullet}(F)$ induces an isomorphism $H^{r}\left(\tau_{\geq k} \Omega^{\bullet} F\right) \stackrel{\cong}{\Longrightarrow} H^{r}(F)$ in the range $r \geq k$. A key advantage of cotruncation over truncation is that $\tau_{\geq k} \Omega^{\circ} F$ is a subalgebra of $\left(\Omega^{\bullet} F, \wedge\right)$, whereas $\tau_{<k} \Omega^{\bullet} F$ is not.

Proposition 3.3. The isomorphism type of $\tau_{\geq k} \Omega^{\bullet} F$ in the category of cochain complexes is independent of the Riemannian metric on $F$.

Lemma 3.4. Let $f: F \rightarrow F$ be a smooth self-map.

(1) $f$ induces an endomorphism $f^{*}$ of $\tau_{<k} \Omega^{\bullet} F$.

(2) If $f$ is an isometry, then $f$ induces an automorphism $f^{*}$ of $\tau_{\geq k} \Omega^{\bullet} F$.

We shall next define the fiberwise truncation $\mathrm{ft}_{<t} \Omega_{\mathcal{M S}}^{\bullet}\left(\mathbb{R}^{n}\right) \subset \Omega_{\mathcal{M S}}^{\bullet}\left(\mathbb{R}^{n}\right)$ and the fiberwise cotruncation $\mathrm{ft}_{\geq t} \Omega_{\mathcal{M S}}^{\bullet}\left(\mathbb{R}^{n}\right) \subset \Omega_{\mathcal{M S}}^{\cdot}\left(\mathbb{R}^{n}\right)$, depending on an integer $t$. Set

$$
\begin{aligned}
& \mathrm{ft}_{<t} \Omega_{\mathcal{M S}}^{\bullet}\left(\mathbb{R}^{n}\right) \\
& \quad=\left\{\omega \in \Omega^{\bullet}\left(\mathbb{R}^{n} \times F\right) \mid \omega=\sum_{j} \pi_{1}^{*} \eta_{j} \wedge \pi_{2}^{*} \gamma_{j}, \eta_{j} \in \Omega^{\bullet}\left(\mathbb{R}^{n}\right), \gamma_{j} \in \tau_{<t} \Omega^{\bullet}(F)\right\} .
\end{aligned}
$$

The complex $\mathrm{ft}_{<t} \Omega_{\mathcal{M S}}^{\bullet}\left(\mathbb{R}^{n}\right)$ is a subcomplex of $\Omega_{\mathcal{M S}}^{\bullet}\left(\mathbb{R}^{n}\right)$. Define

$$
\begin{aligned}
& \mathrm{ft}_{\geq t} \Omega_{\mathcal{M S}}^{\bullet}\left(\mathbb{R}^{n}\right) \\
& \quad=\left\{\omega \in \Omega^{\bullet}\left(\mathbb{R}^{n} \times F\right) \mid \omega=\sum_{j} \pi_{1}^{*} \eta_{j} \wedge \pi_{2}^{*} \gamma_{j}, \eta_{j} \in \Omega^{\bullet}\left(\mathbb{R}^{n}\right), \gamma_{j} \in \tau_{\geq t} \Omega^{\bullet}(F)\right\} .
\end{aligned}
$$


Again, this is a subcomplex of $\Omega_{\mathcal{M S}}^{\bullet}\left(\mathbb{R}^{n}\right)$. Similarly, a subcomplex

$$
\mathrm{ft}_{<t} \Omega_{\mathcal{M} S}^{\bullet}\left(U_{\alpha_{0} \ldots \alpha_{p}}\right) \subset \Omega_{\mathcal{M} S}^{\bullet}\left(U_{\alpha_{0} \ldots \alpha_{p}}\right)
$$

of fiberwise truncated multiplicatively structured forms on $\pi^{-1}\left(U_{\alpha_{0} \ldots \alpha_{p}}\right)$ is given by requiring every $\gamma_{j}$ to lie in $\tau_{<t} \Omega^{\bullet}(F)$. This is well defined by the transformation law (1) together with Lemma 3.4 (1). A subcomplex

$$
\mathrm{ft}_{\geq t} \Omega_{\mathcal{M S}}^{\bullet}\left(U_{\alpha_{0} \ldots \alpha_{p}}\right) \subset \Omega_{\mathcal{M} S}^{\bullet}\left(U_{\alpha_{0} \ldots \alpha_{p}}\right)
$$

of fiberwise cotruncated multiplicatively structured forms on $\pi^{-1}\left(U_{\alpha_{0} \ldots \alpha_{p}}\right)$ is given by requiring every $\gamma_{j}$ to lie in $\tau_{\geq t} \Omega^{\bullet}(F)$. This is well defined by the transformation law and Lemma 3.4(2). (At this point, it is used that the transition functions of the bundle are isometries.)

Let $S: \mathbb{R}^{n-1} \times F \hookrightarrow \mathbb{R}^{n} \times F, Q: \mathbb{R}^{n} \times F \rightarrow \mathbb{R}^{n-1} \times F$, be as in Section 2 . The induced map $S^{*}: \Omega_{\mathcal{M S}}^{\bullet}\left(\mathbb{R}^{n}\right) \rightarrow \Omega_{\mathcal{M S}}^{\bullet}\left(\mathbb{R}^{n-1}\right)$ restricts to a map

$$
S^{*}: \mathrm{ft}_{<t} \Omega_{\mathcal{M} S}^{\bullet}\left(\mathbb{R}^{n}\right) \rightarrow \mathrm{ft}_{<t} \Omega_{\mathcal{M} S}^{\bullet}\left(\mathbb{R}^{n-1}\right) .
$$

The induced map $Q^{*}: \Omega_{\mathcal{M} S}^{\bullet}\left(\mathbb{R}^{n-1}\right) \rightarrow \Omega_{\mathcal{M S}}^{\bullet}\left(\mathbb{R}^{n}\right)$ restricts to a map

$$
Q^{*}: \mathrm{ft}_{<t} \Omega_{\mathcal{M} S}^{\bullet}\left(\mathbb{R}^{n-1}\right) \rightarrow \mathrm{ft}_{<t} \Omega_{\mathcal{M} S}^{\bullet}\left(\mathbb{R}^{n}\right)
$$

Lemma 3.5. The maps

$$
\mathrm{ft}_{<t} \Omega_{\mathcal{M} S}^{\bullet}\left(\mathbb{R}^{n}\right) \stackrel{S^{*}}{\underset{Q^{*}}{\rightleftarrows}} \mathrm{ft}_{<t} \Omega_{\mathcal{M} S}^{\bullet}\left(\mathbb{R}^{n-1}\right)
$$

are chain homotopy inverses of each other and thus induce mutually inverse isomorphisms

$$
H^{\bullet}\left(\mathrm{ft}_{<t} \Omega_{\mathcal{M S}}^{\bullet}\left(\mathbb{R}^{n}\right)\right) \underset{Q^{*}}{\stackrel{S^{*}}{\rightleftarrows}} H^{\bullet}\left(\mathrm{ft}_{<t} \Omega_{\mathcal{M S}}^{\bullet}\left(\mathbb{R}^{n-1}\right)\right)
$$

on cohomology.

As in Section 2, let $S_{0}: F=\{0\} \times F \hookrightarrow \mathbb{R}^{n} \times F$ be the inclusion at 0 . The induced map $S_{0}^{*}: \Omega_{\mathcal{M S}}^{\bullet}\left(\mathbb{R}^{n}\right) \rightarrow \Omega^{\bullet}(F)$ restricts to a map $S_{0}^{*}: \mathrm{ft}_{<t} \Omega_{\mathcal{M} S}\left(\mathbb{R}^{n}\right) \rightarrow$ $\tau_{<t} \Omega^{\bullet}(F)$. The map $\pi_{2}^{*}: \Omega^{\bullet}(F) \rightarrow \Omega_{\mathcal{M S}}^{\bullet}\left(\mathbb{R}^{n}\right)$ restricts to a map $\pi_{2}^{*}: \tau_{<t} \Omega^{\bullet}(F) \rightarrow$ $\mathrm{ft}_{<t} \Omega_{\mathcal{M} S}^{\bullet}\left(\mathbb{R}^{n}\right)$. An induction on $n$ using Lemma 3.5 shows:

Lemma 3.6 (Poincaré lemma for truncated forms). The maps

$$
\mathrm{ft}_{<t} \Omega_{\mathcal{M} S}^{\bullet}\left(\mathbb{R}^{n}\right) \underset{\pi_{2}^{*}}{\stackrel{S_{0}^{*}}{\rightleftarrows}} \tau_{<t} \Omega^{\bullet}(F)
$$


are chain homotopy inverses of each other and thus induce mutually inverse isomorphisms

$$
H^{r}\left(\mathrm{ft}_{<t} \Omega_{\mathcal{M S}}^{\bullet}\left(\mathbb{R}^{n}\right)\right) \underset{\pi_{2}^{*}}{\stackrel{S_{0}^{*}}{\rightleftarrows}} H^{r}\left(\tau_{<t} \Omega^{\bullet}(F)\right) \cong \begin{cases}H^{r}(F), & r<t, \\ 0, & r \geq t,\end{cases}
$$

on cohomology.

An analogous argument, replacing $\tau_{<t} \Omega^{\bullet}(F)$ by $\tau_{\geq t} \Omega^{\bullet}(F)$, proves a version for fiberwise cotruncation:

Lemma 3.7 (Poincaré lemma for cotruncated forms). The maps

$$
\mathrm{ft}_{\geq t} \Omega_{\mathcal{M S}}^{\cdot}\left(\mathbb{R}^{n}\right) \underset{\pi_{2}^{*}}{\stackrel{S_{0}^{*}}{\rightleftarrows}} \tau_{\geq t} \Omega^{\bullet}(F)
$$

are chain homotopy inverses of each other and thus induce mutually inverse isomorphisms

$$
H^{r}\left(\mathrm{ft}_{\geq t} \Omega_{\mathcal{M S}}^{\bullet}\left(\mathbb{R}^{n}\right)\right) \underset{\pi_{2}^{*}}{\stackrel{S_{0}^{*}}{\rightleftarrows}} H^{r}\left(\tau_{\geq t} \Omega^{\bullet}(F)\right) \cong \begin{cases}H^{r}(F), & r \geq t, \\ 0, & r<t,\end{cases}
$$

on cohomology.

\section{4. Čech presheaves}

Let $M$ be a smooth manifold and $\mathfrak{V}=\left\{V_{\alpha}\right\}$ be a good open cover of $M$. The cover $\mathfrak{V}$ gives rise to a category $C(\mathfrak{V})$, whose objects are all finite intersections $V_{\alpha_{0} \ldots \alpha_{p}}$ of open sets $V_{\alpha}$ in $\mathfrak{V}$ and an initial object $\varnothing$, the empty set. The morphisms are inclusions. A Čech presheaf $\boldsymbol{H}$ on $\mathfrak{V}$ is a contravariant functor $\boldsymbol{H}: C(\mathfrak{V}) \rightarrow \mathbb{R}-\mathrm{MOD}$ into the category $\mathbb{R}-\mathrm{MOD}$ of real vector spaces and linear maps, such that $\boldsymbol{H}(\varnothing)=0$. A homomorphism $\boldsymbol{H} \rightarrow \boldsymbol{G}$ of Čech presheaves on $\mathfrak{V}$ is a natural transformation from $\boldsymbol{H}$ to $\boldsymbol{G}$. The homomorphism is an isomorphism if $\boldsymbol{H}\left(V_{\alpha_{0} \ldots \alpha_{p}}\right) \rightarrow \boldsymbol{G}\left(V_{\alpha_{0} \ldots \alpha_{p}}\right)$ is an isomorphism for every object $V_{\alpha_{0} \ldots \alpha_{p}}$ in $C(\mathfrak{V})$. Let $H$ be a real vector space. The presheaf $\boldsymbol{H}$ is said to be locally constant with group $H$ if all $\boldsymbol{H}\left(V_{\alpha_{0} \ldots \alpha_{p}}\right)$, for $V_{\alpha_{0} \ldots \alpha_{p}} \neq \varnothing$, are isomorphic to $H$ and all linear maps $\boldsymbol{H}\left(V_{\alpha_{0} \ldots \alpha_{p}}\right) \rightarrow \boldsymbol{H}\left(V_{\beta_{0} \ldots \beta_{q}}\right)$ for nonempty inclusions $V_{\beta_{0} \ldots \beta_{q}} \subset V_{\alpha_{0} \ldots \alpha_{p}}$ are isomorphisms. A Čech presheaf $\boldsymbol{H}$ on $\mathfrak{V}$ possesses $p$-cochains

$$
C^{p}(\mathfrak{V} ; \boldsymbol{H})=\prod_{\alpha_{0}, \ldots, \alpha_{p}} \boldsymbol{H}\left(V_{\alpha_{0} \ldots \alpha_{p}}\right)
$$


and a Čech differential $\delta: C^{p}(\mathfrak{V} ; \boldsymbol{H}) \rightarrow C^{p+1}(\mathfrak{V} ; \boldsymbol{H})$ making $C^{\bullet}(\mathfrak{V} ; \boldsymbol{H})$ into a complex. Its cohomology $H^{p}(\mathfrak{V} ; \boldsymbol{H})$ is the Čech cohomology of the cover $\mathfrak{V}$ with values in $\boldsymbol{H}$. An isomorphism $\boldsymbol{H} \cong \boldsymbol{G}$ of two presheaves on $\mathfrak{V}$ induces an isomorphism of cohomology groups $H^{\bullet}(\mathfrak{V} ; \boldsymbol{H}) \stackrel{\cong}{\longrightarrow} H^{\bullet}(\mathfrak{V} ; \boldsymbol{G})$. Let $\boldsymbol{L}$ be a locally constant sheaf on $M$. Then $\boldsymbol{L}$ defines in particular a locally constant presheaf on $\mathfrak{V}$ (since $\mathfrak{V}$ is good, $\left.\boldsymbol{L}\right|_{V}$ is constant for every $V \in \mathrm{Ob} C(\mathfrak{V})$ ) so that $H^{\bullet}(\mathfrak{V} ; \boldsymbol{L})$ is defined. Then, as $H^{q}\left(V_{\alpha_{0} \ldots \alpha_{p}} ; \boldsymbol{L}\right)=0$ for $q>0$, there is a canonical isomorphism $H_{\mathrm{Sh}}^{\bullet}(M ; \boldsymbol{L}) \cong H^{\bullet}(\mathfrak{V} ; \boldsymbol{L})$ according to [5], Thm. III.4.13, where $H_{\mathrm{Sh}}^{\bullet}(M ; \boldsymbol{L})$ denotes sheaf cohomology. In particular, the Čech cohomology groups of $\mathfrak{V}$ with values in $\boldsymbol{L}$ are independent of the good cover used to define them. By [5], Cor. III.4.12, these groups are furthermore canonically isomorphic to the Čech cohomology of $M$ with coefficients in $\boldsymbol{L}, \check{H}^{\bullet}(M ; \boldsymbol{L})$. If we view $\boldsymbol{L}$ as a local coefficient system on $M$, then the singular cohomology $H^{\bullet}(M ; \boldsymbol{L})$ is defined and a canonical isomorphism $H_{\text {Sh }}^{\bullet}(M ; \boldsymbol{L}) \cong H^{\bullet}(M ; \boldsymbol{L})$ is provided by [5], Thm. III.1.1.

Let us return to the good cover $\mathfrak{U}$ on our base space $B$. We shall define three Čech presheaves on $\mathcal{U}$. Define $\boldsymbol{H}^{q}(F)$ by

$$
\boldsymbol{H}^{q}(F)(U)=H^{q}\left(\pi^{-1} U\right), \quad U \in \mathrm{Ob} C(\mathfrak{U}) .
$$

The structural morphisms associated to inclusions are given by restriction of forms. Since all nonempty objects $U$ in $C(\mathfrak{U})$ are diffeomorphic to $\mathbb{R}^{n}$, and the bundle $\pi: E \rightarrow B$ trivializes over every such $U$, the classical Poincaré lemma implies that $\boldsymbol{H}^{q}(F)$ is a locally constant presheaf with group $H^{q}(F)$, the de Rham cohomology of the fiber. Define the presheaf $\boldsymbol{H}_{\mathcal{M S}}^{q}(F)$ by

$$
\boldsymbol{H}_{\mathcal{M S}}^{q}(F)(U)=H^{q}\left(\Omega_{\mathcal{M S}}^{\bullet}(U)\right), \quad U \in \mathrm{Ob} C(\mathfrak{U}) .
$$

According to Proposition 2.10, the inclusion $\Omega_{\mathcal{M S}}^{\bullet}(U) \subset \Omega^{\bullet}\left(\pi^{-1} U\right)$ induces an isomorphism $\boldsymbol{H}_{\mathcal{M S}}^{q}(F)(U) \stackrel{\cong}{\cong} \boldsymbol{H}^{q}(F)(U)$ for every nonempty $U \in \mathrm{Ob} C(\mathfrak{U})$. If $V \in \mathrm{Ob} C(\mathfrak{U})$ is an open set with $V \subset U$, then the commutative square

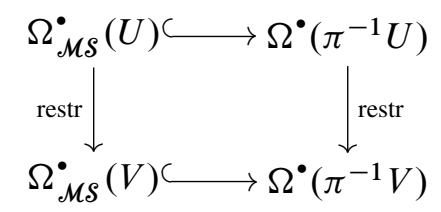

induces a commutative square

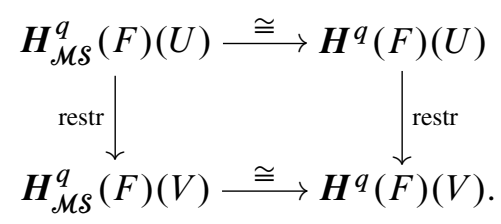

Thus the inclusion of multiplicatively structured forms induces an isomorphism

$$
\boldsymbol{H}_{\mathcal{M S}}^{q}(F) \stackrel{\cong}{\longrightarrow} \boldsymbol{H}^{q}(F)
$$


of presheaves for every $q$. In particular, $\boldsymbol{H}_{\mathcal{M S}}^{q}(F)$ is also locally constant with group $H^{q}(F)$. The isomorphism induces furthermore an isomorphism

$$
H^{p}\left(\mathfrak{U} ; \boldsymbol{H}_{\mathcal{M S}}^{q}(F)\right) \stackrel{\cong}{\cong} H^{p}\left(\mathfrak{U} ; \boldsymbol{H}^{q}(F)\right)
$$

of Čech cohomology groups. Define the presheaf $\boldsymbol{H}_{\geq t}^{q}(F)$ by

$$
\boldsymbol{H}_{\geq t}^{q}(F)(U)=H^{q}\left(\mathrm{ft}_{\geq t} \Omega_{\mathcal{M S}}^{\bullet}(U)\right), \quad U \in \mathrm{Ob} C(\mathfrak{U}), U \neq \varnothing .
$$

Since $U \neq \varnothing$ is diffeomorphic to $\mathbb{R}^{n}$ and the bundle $\pi: E \rightarrow B$ trivializes over $U$, the Poincaré Lemma 3.7 for cotruncated forms implies that the restriction $S_{0}^{*}$ of a form to the fiber over the origin of $U \cong \mathbb{R}^{n}$ induces an isomorphism

$$
\boldsymbol{H}_{\geq t}^{q}(F)(U) \underset{S_{0}^{*}}{\stackrel{\cong}{\longrightarrow}} H^{q}(F)
$$

for $q \geq t$, whereas $\boldsymbol{H}_{\geq t}^{q}(F)(U)=0$ for $q<t$. If $V \in \mathrm{ObC}(\mathfrak{U})$ is a nonempty open set with $V \subset U$, then the commutative diagram

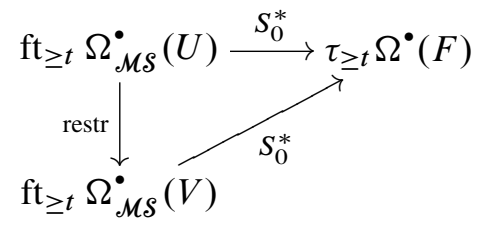

(assuming, without loss of generality, that the origin of $U$ lies in $V$ ) induces a commutative diagram

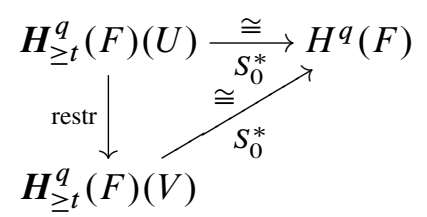

for $q \geq t$. The restriction induces an isomorphism $\boldsymbol{H}_{\geq t}^{q}(F)(U) \cong \boldsymbol{H}_{\geq t}^{q}(F)(V)$. Therefore, when $q \geq t, \boldsymbol{H}_{\geq t}^{q}(F)$ is a locally constant presheaf with group $H^{q}(F)$. Moreover, the commutative diagram

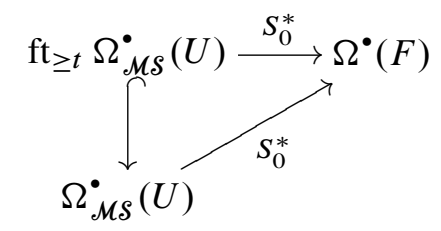

induces, for $q \geq t$, a commutative diagram

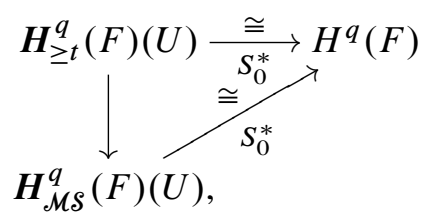


using Proposition 2.8. Thus $\boldsymbol{H}_{\geq t}^{q}(F)(U) \rightarrow \boldsymbol{H}_{\mathcal{M} S}^{q}(F)(U)$ is an isomorphism for $q \geq t$. Since it commutes with restriction to smaller open sets, we obtain the following result.

Lemma 4.1. For $q<t$, the presheaf $\boldsymbol{H}_{\geq t}^{q}(F)$ is trivial, $\boldsymbol{H}_{\geq t}^{q}(F)=0$. For $q \geq t$, $\boldsymbol{H}_{\geq t}^{q}(F)$ is locally constant with group $H^{q}(F)$ and the inclusion $\mathrm{ft}_{\geq t} \Omega_{\mathcal{M S}}^{\bullet}(-) \subset$ $\Omega_{\mathcal{M S}}^{-}(-)$induces an isomorphism

$$
\boldsymbol{H}_{\geq t}^{q}(F) \stackrel{\cong}{\longrightarrow} \boldsymbol{H}_{\mathcal{M} S}^{q}(F)
$$

of presheaves.

\section{The spectral sequence of a flat, isometrically structured bundle}

Let $(K, \delta, d)$ be the double complex

$$
K^{p, q}=C^{p}\left(\pi^{-1} \mathfrak{U} ; \Omega^{q}\right)=\prod_{\alpha_{0}, \ldots, \alpha_{p}} \Omega^{q}\left(\pi^{-1} U_{\alpha_{0} \ldots \alpha_{p}}\right)
$$

defined in Section 2. The spectral sequence of the fiber bundle $\pi: E \rightarrow B$ is the spectral sequence $E(K)=\left\{E_{r}, d_{r}\right\}$ of $K$. Its $E_{1}$-term is

$$
E_{1}^{p, q}=H_{d}^{p, q}(K)=\prod_{\alpha_{0}, \ldots, \alpha_{p}} H^{q}\left(\pi^{-1} U_{\alpha_{0} \ldots \alpha_{p}}\right)=C^{p}\left(\mathfrak{U} ; \boldsymbol{H}^{q}(F)\right) .
$$

Since $d_{1}=\delta$ on $E_{1}$, the $E_{2}$-term is

$$
E_{2}^{p, q}=H^{p}\left(\mathfrak{U} ; \boldsymbol{H}^{q}(F)\right) .
$$

Let $\mathscr{H}^{q}(F)$ be the Leray sheaf of $\pi: E \rightarrow B$, that is, the higher direct image sheaf $R^{q} \pi_{*} \mathbb{R}_{E}$, where $\mathbb{R}_{E}$ denotes the constant sheaf with stalk $\mathbb{R}$ on $E$. The Leray sheaf $\mathscr{H}^{q}(F)$ is the sheafification of the presheaf $A$ on $B$ given by $A(U)=H^{q}\left(\pi^{-1} U\right)$, $U \subset B$ open ([8], Prop. III.8.1). Let $F_{b}=\pi^{-1}(\{b\})$ denote the fiber over a point $b \in B$. The restriction maps $H^{\bullet}\left(\pi^{-1} U\right) \rightarrow H^{\bullet}\left(F_{u}\right), u \in U$, induce an isomorphism

$$
\mathcal{H}^{\bullet}(F)_{u} \stackrel{\cong}{\cong} H^{\bullet}\left(F_{u}\right),
$$

where $\mathscr{H}^{\bullet}(F)_{u}$ is the stalk of $\mathscr{H}^{\bullet}(F)$ at $u$, [5], Prop. IV.4.2. By [5], Cor. IV.7.3, $\mathscr{H}^{q}(F)$ is locally constant. As explained in Section 4, a locally constant sheaf $\mathscr{H}^{q}(F)$ determines a locally constant Čech presheaf of sections $\mathscr{H}^{q}(F) \mathfrak{u}=\Gamma\left(-; \mathscr{H}^{q}(F)\right)$ on the cover $\mathfrak{U}$ such that

$$
H_{\mathrm{Sh}}^{\bullet}\left(B ; \mathscr{H}^{q}(F)\right) \cong H^{\bullet}\left(\mathfrak{U} ; \mathscr{H}^{q}(F) \mathfrak{U}\right) .
$$


Lemma 5.1. The canonical map $\boldsymbol{H}^{q}(F)(U) \rightarrow \Gamma\left(U ; \mathscr{H}^{q}(F)\right), U \in \mathrm{Ob} C(\mathfrak{U})$, given by sending a cohomology class $x \in H^{q}\left(\pi^{-1} U\right)$ to the section $u \mapsto x_{u}, u \in U$ (where $x_{u}$ is the germ of $x$ at $u$ ), defines an isomorphism $\boldsymbol{H}^{q}(F) \cong \mathscr{H}^{q}(F)_{\mathfrak{u}}$ of Čech presheaves on $\mathfrak{U}$.

Proof. The diagram

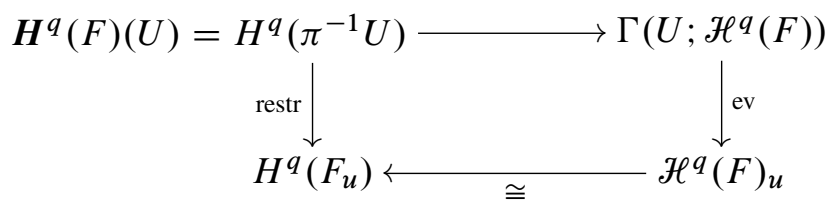

commutes, where the right hand vertical map is evaluation, sending a section over $U$ to its value at $u$. For nonempty $U \in \mathrm{Ob} C(\mathfrak{U}), \pi^{-1}(U) \cong U \times F \cong \mathbb{R}^{n} \times F$ and the left hand vertical restriction map is an isomorphism, e.g. by the Künneth theorem. The evaluation map is an isomorphism because $\mathscr{H}^{q}(F)$ is locally constant and thus constant on $U \cong \mathbb{R}^{n}$. Thus $\boldsymbol{H}^{q}(F)(U) \rightarrow \Gamma\left(U ; \mathscr{H}^{q}(F)\right)$ is an isomorphism for every $U \in \mathrm{Ob} C(\mathfrak{H})$.

(We do not claim, of course, that there is an isomorphism $A \cong \Gamma\left(-; \mathscr{H}^{q}(F)\right.$ ) of presheaves on $B$.) Using this lemma and [5], Thm. III.1.1, the $E_{2}$-term can be identified as

$$
\begin{aligned}
E_{2}^{p, q}=H^{p}\left(\mathfrak{U} ; \boldsymbol{H}^{q}(F)\right) & \cong H^{p}\left(\mathfrak{U} ; \mathscr{H}^{q}(F) \mathfrak{U}\right) \\
& \cong H_{\mathrm{Sh}}^{p}\left(B ; \mathscr{H}^{q}(F)\right) \cong H^{p}\left(B ; \mathscr{H}^{q}(F)\right),
\end{aligned}
$$

where in the last group, the singular cohomology group, we have interpreted the Leray sheaf as a local coefficient system.

Theorem 5.2. Let $F$ be a closed, oriented, Riemannian manifold and $\pi: E \rightarrow B a$ flat, smooth fiber bundle over the closed, smooth base manifold $B$ with fiber $F$ and structure group the isometries of $F$. Then the spectral sequence with real coefficients of $\pi: E \rightarrow B$ collapses at the $E_{2}$-term.

Proof. Using multiplicatively structured forms, we first build a smaller model $K_{\mathcal{M S}}$ of $K$. The spectral sequences of $K_{\mathcal{M} S}$ and $K$ will be shown to be isomorphic (from the $E_{2}$-term on). In Section 2, we introduced the multiplicatively structured Čech-de Rham double complex $K_{\mathcal{M S}}=\left(C^{\bullet}\left(\mathfrak{U} ; \Omega_{\mathcal{M S}}^{\bullet}\right), \delta, d\right)$. In bidegree $(p, q)$ it is given by

$$
K_{\mathcal{M S}}^{p, q}=C^{p}\left(\mathfrak{U} ; \Omega_{\mathcal{M S}}^{q}\right)=\prod_{\alpha_{0}, \ldots, \alpha_{p}} \Omega_{\mathcal{M S}}^{q}\left(U_{\alpha_{0} \ldots \alpha_{p}}\right) .
$$

The vertical cohomology of $K_{\mathcal{M} S}$ is

$$
H_{d}^{p, q}\left(K_{\mathcal{M S}}\right)=\prod_{\alpha_{0}, \ldots, \alpha_{p}} H^{q}\left(\Omega_{\mathcal{M S}}^{\bullet}\left(U_{\alpha_{0} \ldots \alpha_{p}}\right)\right)=C^{p}\left(\mathfrak{U} ; \boldsymbol{H}_{\mathcal{M S}}^{q}(F)\right) .
$$


The core of the argument is the construction of a filtration of $K_{\mathcal{M S}}$ by cotruncated double complexes $K_{\geq t} \subset K_{\mathcal{M S}}$, where $t$ is an integer. The group in bidegree $(p, q)$ is

$$
K_{\geq t}^{p, q}=C^{p}\left(\mathfrak{U} ;\left(\mathrm{ft}_{\geq t} \Omega_{\mathcal{M S}}^{\bullet}\right)^{q}\right)=\prod_{\alpha_{0}, \ldots, \alpha_{p}}\left(\mathrm{ft}_{\geq t} \Omega_{\mathcal{M S}}^{\bullet}\left(U_{\alpha_{0} \ldots \alpha_{p}}\right)\right)^{q} .
$$

The vertical differential is given by the (restriction of the) de Rham differential $d$, and the horizontal differential is given by the Čech differential $\delta$. The vertical cohomology of $K_{\geq t}$ is

$$
H_{d}^{p, q}\left(K_{\geq t}\right)=\prod_{\alpha_{0}, \ldots, \alpha_{p}} H^{q}\left(\mathrm{ft}_{\geq t} \Omega_{\mathcal{M S}}^{\bullet}\left(U_{\alpha_{0} \ldots \alpha_{p}}\right)\right)=C^{p}\left(\mathfrak{U} ; \boldsymbol{H}_{\geq t}^{q}(F)\right) .
$$

The double complex $K_{\mathcal{M S}}$ determines a spectral sequence

$$
E\left(K_{\mathcal{M} S}\right)=\left\{E_{\mathcal{M} S, r}, d_{\mathcal{M} S, r}\right\} ;
$$

the double complex $K_{\geq t}$ determines a spectral sequence

$$
E\left(K_{\geq t}\right)=\left\{E_{\geq t, r}, d_{\geq t, r}\right\},
$$

cf. [4], Thm. 14.14, p. 165. The inclusions of complexes

$$
\mathrm{ft}_{\geq t} \Omega_{\mathcal{M S}}^{\bullet}(U) \subset \Omega_{\mathcal{M S}}^{\bullet}(U) \subset \Omega^{\bullet}\left(\pi^{-1} U\right), \quad U \in \mathrm{Ob} C(\mathfrak{U}),
$$

induce inclusions of double complexes

$$
K_{\geq t} \subset K_{\mathcal{M S}} \subset K .
$$

A map of double complexes induces a morphism of the associated spectral sequences. Thus the above inclusions induce morphisms

$$
E\left(K_{\geq t}\right) \stackrel{e}{\rightarrow} E\left(K_{\mathcal{M S}}\right) \stackrel{f}{\rightarrow} E(K) .
$$

Let us show that the differentials $d_{\mathcal{M} S, 2}$ vanish. This will then provide the induction basis for an inductive proof that all $d_{\mathcal{M} S, r}, r \geq 2$, vanish. The term $E_{\mathcal{M S}, 1}$ is given by

$$
E_{\mathcal{M} S, 1}^{p, q}=H_{d}^{p, q}\left(K_{\mathcal{M} S}\right)=C^{p}\left(\mathfrak{U} ; \boldsymbol{H}_{\mathcal{M} S}^{q}(F)\right) .
$$

Since $d_{\mathcal{M} S, 1}=\delta$, we have

$$
E_{\mathcal{M S}, 2}^{p, q}=H_{\delta}^{p}\left(\mathcal{U} ; \boldsymbol{H}_{\mathcal{M} S}^{q}(F)\right),
$$

the Čech cohomology of $\mathcal{U}$ with values in the presheaf $\boldsymbol{H}_{\mathcal{M S}}^{q}(F)$. The term $E_{\geq t, 1}$ is given by

$$
E_{\geq t, 1}^{p, q}=H_{d}^{p, q}\left(K_{\geq t}\right)=C^{p}\left(\mathfrak{U} ; \boldsymbol{H}_{\geq t}^{q}(F)\right) .
$$


Since $d_{\geq t, 1}=\delta$, we have

$$
E_{\geq t, 2}^{p, q}=H_{\delta}^{p}\left(\mathfrak{U} ; \boldsymbol{H}_{\geq t}^{q}(F)\right) .
$$

Set $t=q$. Since $e$ is a morphism of spectral sequences, we have a commutative square

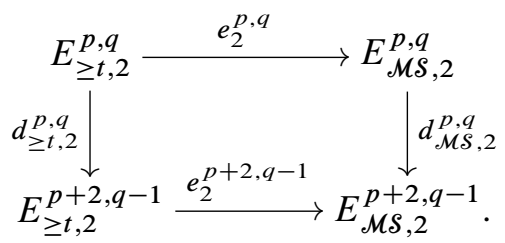

In view of the above identifications of $E_{2}$-terms, this can be rewritten as

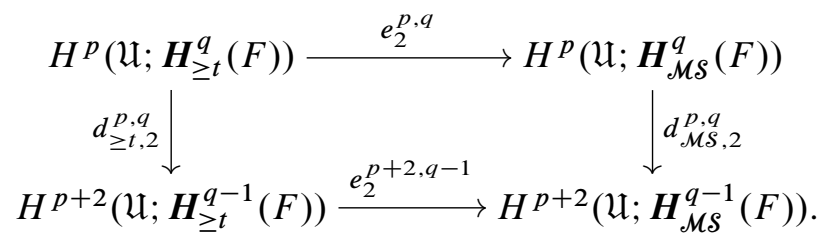

Our choice of $t$ together with Lemma 4.1 implies that $e_{2}^{p, q}$ is an isomorphism. Therefore, we can express $d_{\mathcal{M} \mathcal{S}, 2}^{p, q}$ as the composition

$$
d_{\mathcal{M S}, 2}^{p, q}=e_{2}^{p+2, q-1} \circ d_{\geq t, 2}^{p, q} \circ\left(e_{2}^{p, q}\right)^{-1} .
$$

As $q-1<t$, we have $\boldsymbol{H}_{\geq t}^{q-1}(F)=0$ by Lemma 4.1, and so $H^{p+2}\left(\mathfrak{U} ; \boldsymbol{H}_{\geq t}^{q-1}(F)\right)=$ $0, d_{\geq t, 2}^{p, q}=0$ and $e_{2}^{p+2, q-1}=0$. By (3), $d_{\mathcal{M} s, 2}^{p, q}=0$.

We shall next show that for arbitrary $t, d_{\geq t, 2}=0$. Given any bidegree $(p, q)$, there are two cases to consider: $q-1<t$ and $q-1 \geq t$. If $q-1<t$, then we have $E_{\geq t, 2}^{p+q-1}=H^{p+2}\left(\mathfrak{U} ; \boldsymbol{H}_{\geq t}^{q-1}(F)\right)=0$, so that $d_{\geq t, 2}^{p, q}: E_{\geq t, 2}^{p, q} \rightarrow E_{\geq t, 2}^{p+2, q-1}=0$ is zero. If $q-1 \geq t$, then $e_{2}^{p+2, q-1}$ is an isomorphism by Lemma 4.1 and

$$
d_{\geq t, 2}^{p, q}=\left(e_{2}^{p+2, q-1}\right)^{-1} \circ d_{\mathcal{M} s, 2}^{p, q} \circ e_{2}^{p, q}=0
$$

since $d_{\mathcal{M} s, 2}^{p, q}=0$. Thus $d_{\geq t, 2}=0$ for any $t$.

For $r \geq 2$, let $\mathrm{P}(r)$ denote the package of statements

- $d_{\mathcal{M} S, r}=0$ and $d_{\geq t, r}=0$ for all $t$,

- $E_{\mathcal{M} S, r}^{p, q}=H^{p}\left(\mathcal{U} ; \boldsymbol{H}_{\mathcal{M} S}^{q}(F)\right)$ and $E_{\geq t, r}^{p, q}=H^{p}\left(\mathfrak{U} ; \boldsymbol{H}_{\geq t}^{q}(F)\right)$ for all $t$, and

- $e_{r}=e_{2}$.

We have shown that $\mathrm{P}(2)$ holds. We shall now show for $r \geq 3$ that if $\mathrm{P}(r-1)$ holds, then $\mathrm{P}(r)$ holds. The vanishing of the differentials in the $E_{r-1}$-terms implies that

$$
E_{\mathcal{M} \delta, r}^{p, q}=E_{\mathcal{M} S, r-1}^{p, q}=H^{p}\left(\mathfrak{U} ; \boldsymbol{H}_{\mathcal{M} \mathcal{S}}^{q}(F)\right)
$$


and

$$
E_{\geq t, r}^{p, q}=E_{\geq t, r-1}^{p, q}=H^{p}\left(\mathcal{U} ; \boldsymbol{H}_{\geq t}^{q}(F)\right) .
$$

Furthermore, as $e$ is a morphism of spectral sequences, we have

$$
e_{r}^{p, q}=H^{p, q}\left(e_{r-1}\right)=e_{r-1}^{p, q}=e_{2}^{p, q} .
$$

Hence the commutative square

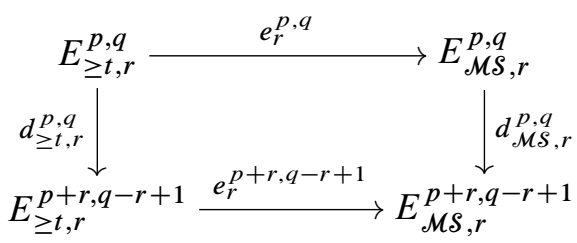

can be rewritten as

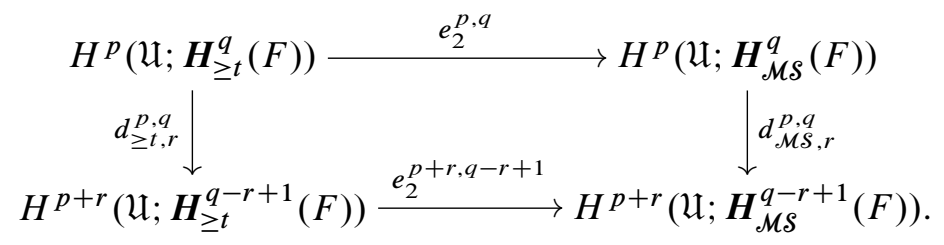

Again take $t=q$. Then $e_{2}^{p, q}$ is an isomorphism and the factorization

$$
d_{\mathcal{M} S, r}^{p, q}=e_{2}^{p+r, q-r+1} \circ d_{\geq t, r}^{p, q} \circ\left(e_{2}^{p, q}\right)^{-1}
$$

shows that $d_{\mathcal{M} s, r}^{p, q}=0$ because $H^{p+r}\left(\mathfrak{U} ; \boldsymbol{H}_{\geq t}^{q-r+1}(F)\right)=0$ by $q-r+1<t$ $(r \geq 3)$. For arbitrary $t, d_{\geq t, r}=0$. For if $q-r+1<t$, then $E_{\geq t, r}^{p+r, q-r+1}=$ $H^{p+r}\left(\mathfrak{U} ; \boldsymbol{H}_{\geq t}^{q-r+1}(F)\right)=0$ so that $d_{\geq t, r}^{p, q}=0$, while for $q-r+1 \geq t$, the map $e_{2}^{p+r, q-r+1}$ is an isomorphism and $d_{\geq t, r}^{p, q}=0$ follows from the factorization $d_{\geq t, r}^{p, q}=\left(e_{2}^{p+r, q-r+1}\right)^{-1} \circ d_{\mathcal{M S}, r}^{p, q} \circ e_{2}^{p, q}$ and the fact that $d_{\mathcal{M S}, r}=0$. This induction shows that $d_{\mathcal{M S}, r}=0$ for all $r \geq 2$. We conclude that $E\left(K_{\mathcal{M S} S}\right)$ collapses at the $E_{2}$-term. This will now be used to prove that $E(K)$ collapses at $E_{2}$.

Since $f$ is a morphism of spectral sequences, we have for every $(p, q)$ a commutative square

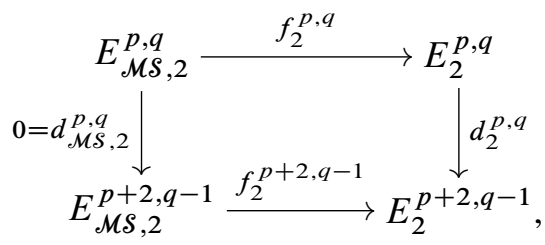


which can be rewritten as

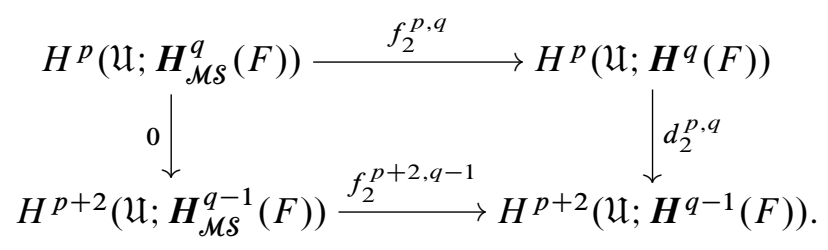

By (2), $f_{2}^{p, q}$ is an isomorphism for all $(p, q)$. Thus $d_{2}=0$ on $E_{2}$. The fact that $f_{2}$ is an isomorphism implies that $f_{r}$ is an isomorphism for all $r \geq 2$. This shows, since $d_{\mathcal{M S}, r}=0$ for all $r \geq 2$, that $d_{r}^{p, q}=0$ for all $r \geq 2$. Consequently, $E(K)$ collapses at the $E_{2}$-term, as was to be shown.

\section{Nonisometrically structured flat bundles}

We construct an example of a flat, smooth circle bundle whose Leray-Serre spectral sequence with real coefficients does not collapse at the $E_{2}$-term. The example shows, then, that in Theorem 5.2 one cannot delete without substitution the requirement that the structure group act isometrically. The example is based on constructions of J. Milnor, cf. [12], [13].

Let $B$ be a closed Riemann surface of genus 2. Its universal cover $\widetilde{B}$ is conformally diffeomorphic to the complex upper half plane $H=\{z \in \mathbb{C} \mid \operatorname{Im} z>0\}$. The fundamental group $\pi_{1}(B)$ acts on $H$ biholomorphically as the deck transformations. The group of biholomorphic automorphisms of $H$ is $\operatorname{PSL}(2, \mathbb{R})$, acting as Möbius transformations

$$
z \mapsto \frac{a z+b}{c z+d}, \quad a, b, c, d \in \mathbb{R}, a d-b c=1 .
$$

This yields a faithful representation $\pi_{1} B \rightarrow \operatorname{PSL}(2, \mathbb{R})$. The operation of $\operatorname{PSL}(2, \mathbb{R})$ on $H$ extends naturally to the closure $\bar{H}=H \cup \mathbb{R} \cup\{\infty\}$ of $H$ in $\mathbb{C} \cup\{\infty\}$. In particular, $\operatorname{PSL}(2, \mathbb{R})$ acts on the boundary circle $\partial \bar{H}=\mathbb{R} \cup\{\infty\}$ and we can form the flat circle bundle $\gamma$ with projection $\pi: E=\widetilde{B} \times_{\pi_{1} B}(\mathbb{R} \cup\{\infty\}) \rightarrow B$ and structure group $\operatorname{PSL}(2, \mathbb{R})$. Recall that any orientable, possibly nonlinear, sphere bundle $\xi$ with structure group Diff $\left(S^{n-1}\right)$ has a real Euler class $e(\xi) \in H^{n}(M ; \mathbb{R})$, where $M$ is the base manifold. Since our bundle $\gamma$ can be identified (though not linearly) with the tangent circle bundle $S(T B)$ of $B$ (see [13]), the Euler number $\langle e(\gamma),[B]\rangle$ of $\gamma$ is the Euler characteristic $\chi(B)=-2$. As the Euler class is transgressive and not zero, the differential $d_{2}: E_{2}^{0,1} \rightarrow E_{2}^{2,0}$ is nontrivial and the spectral sequence of $\gamma$ does not collapse at $E_{2}$. In more detail, let $\sigma \in E_{1}^{0,1}=C^{0}\left(\mathfrak{U} ; \boldsymbol{H}^{1}\left(S^{1}\right)\right)$ be the element corresponding to the usual angular forms on $E$ ([4], Remark 14.20). Since $\gamma$ is orientable, $d_{1}(\sigma)=\delta(\sigma)=0$ and $\sigma$ determines a class $[\sigma] \in E_{2}^{0,1}=$ 
$H^{0}\left(B ; \boldsymbol{H}^{1}\left(S^{1}\right)\right)$. We have $e(\gamma)= \pm d_{2}[\sigma]$ for the transgression

$$
d_{2}: E_{2}^{0,1}=H^{0}\left(B ; \boldsymbol{H}^{1}\left(S^{1}\right)\right) \rightarrow E_{2}^{2,0}=H^{2}\left(B ; \boldsymbol{H}^{0}\left(S^{1}\right)\right)=H^{2}(B ; \mathbb{R}) .
$$

It follows from Theorem 5.2 that there is no Riemannian metric on $S^{1}=\mathbb{R} \cup\{\infty\}$ such that the action of $\pi_{1}(B)$ on $\mathbb{R} \cup\{\infty\}$ is isometric. This statement will now be affirmed directly, without appealing to the theorem.

Proposition 6.1. There is no Riemannian metric on $\mathbb{R} \cup\{\infty\}$ such that the Fuchsian group given by the image of the representation $\pi_{1} B \rightarrow \operatorname{PSL}(2, \mathbb{R})$ acts isometrically on $\mathbb{R} \cup\{\infty\}$.

Proof. By contradiction; suppose there were such a metric. The image $\Gamma \subset \operatorname{PSL}(2, \mathbb{R})$ of the faithful representation $\pi_{1} B \rightarrow \operatorname{PSL}(2, \mathbb{R})$ is a cocompact surface Fuchsian group and hence all nontrivial elements of $\Gamma$ are hyperbolic, that is, $|\operatorname{tr} X|>2$ for $X \in \Gamma-\{1\}$. Let $X$ be such an element. Any hyperbolic element of PSL $(2, \mathbb{R})$ has precisely two fixed points in $\bar{H}$, both of which lie in $\mathbb{R} \cup\{\infty\}$. In particular, $X$ has a finite, real fixed point $x$. Let $v \in T_{x}(\mathbb{R} \cup\{\infty\})$ be any nonzero tangent vector at this fixed point. If

$$
X_{*}: T_{x}(\mathbb{R} \cup\{\infty\}) \rightarrow T_{X(x)}(\mathbb{R} \cup\{\infty\})=T_{x}(\mathbb{R} \cup\{\infty\})
$$

denotes the differential of $X: \mathbb{R} \cup\{\infty\} \rightarrow \mathbb{R} \cup\{\infty\}$ at $x$, then $X_{*}(v)=\lambda v$ for some nonzero scalar $\lambda \in \mathbb{R}$ and

$$
|\lambda| \cdot\|v\|_{x}=\|\lambda v\|_{x}=\left\|X_{*}(v)\right\|_{x}=\|v\|_{x},
$$

where $\|\cdot\|_{x}$ is the putative $\Gamma$-invariant norm, evaluated at $x$. Consequently, $X_{*}(v)=$ $\pm v$. Let $t$ be the standard coordinate in $\mathbb{R} \subset \mathbb{R} \cup\{\infty\}$ and write

$$
X(t)=\frac{a t+b}{c t+d}, \quad a, b, c, d \in \mathbb{R}, a d-b c=1,|a+d|>2 .
$$

Taking $v$ to be the standard basis vector $v=\partial_{t}$, we have $X_{*}\left(\partial_{t}\right)=\frac{d X}{d t}(x) \cdot \partial_{t}$ and thus

$$
\left|\frac{d X}{d t}(x)\right|=1 \text {. }
$$

In fact, however, the derivative of a hyperbolic Möbius transformation in $\operatorname{PSL}(2, \mathbb{R})$ at a finite, real fixed point is never \pm 1 . Indeed, if $c \neq 0$, then

$$
x=\frac{1}{2 c}(a-d \pm \sqrt{\Delta}),
$$

where $\Delta$ is the discriminant $\Delta=\operatorname{tr}^{2} X-4>0$, and if $c=0$, then $x=-b /(a-d)$. (Note that $c=0$ and $X$ hyperbolic implies that $a \neq d$.) The derivative of $X$ is given by $d X / d t=(c t+d)^{-2}$. Brief calculations verify that $(c x+d)^{2}$ cannot be 1 at the above points $x$, for hyperbolic $X$. 
This example illustrates that when modifying the structure group of a fiber bundle, there is a tension between flatness and compactness of the structure group: For a flat bundle with noncompact structure group, one can often reduce to a compact group, but in doing so may be forced to give up flatness. Conversely, given a compactly structured bundle which is not flat, one may sometimes gain flatness at the expense of enlarging the structure group to a noncompact group. For example, identifying the flat $\operatorname{PSL}(2, \mathbb{R})$-bundle $\gamma$ with $S(T B)$, one may give $\gamma$ the structure group $\operatorname{SO}(2)$, but one loses flatness $(S(T B)$ is not a flat $\mathrm{SO}(2)$-bundle).

\section{Equivariant cohomology}

We turn our attention to isometric actions of discrete, torsion-free groups. The actions considered here are usually not proper, and our $G$-spaces are generally not $G$-CW complexes. Concerning condition (1) of Theorem 7.1 below, we remark again that a nonproper action factors in many geometric situations through a proper action; for instance, a discrete, torsion-free group may act nonproperly on a closed manifold, but in such a way that the manifold can be endowed with an invariant Riemannian metric - in that case, the action factors through the (proper) action of the compact isometry group.

Theorem 7.1. Let $F$ be an oriented, closed, smooth manifold and $G$ a discrete group, whose Eilenberg-MacLane space $K(G, 1)$ may be taken to be a closed, smooth manifold. If for a smooth action of $G$ on $F$,

(1) the action factors through the proper, smooth action of a Lie group, or

(2) $F$ is Riemannian and $G$ acts isometrically on $F$,

then the real $G$-equivariant cohomology of $F$ decomposes as

$$
H_{G}^{k}(F ; \mathbb{R}) \cong \bigoplus_{p+q=k} H^{p}\left(G ; \boldsymbol{H}^{q}(F ; \mathbb{R})\right),
$$

where the $\boldsymbol{H}^{q}(F ; \mathbb{R})$ are $G$-modules determined by the action.

Proof. If (1) is satisfied, that is, the action of $G$ on $F$ factors as $G \rightarrow H \rightarrow \operatorname{Diffeo}(F)$ with $H$ a Lie group acting properly, then $F$ can be equipped with an $H$-invariant Riemannian metric, by [15]. Then $H$, and thus also $G$, acts isometrically on $F$ so that it suffices to prove the theorem assuming hypothesis (2). Let $B \simeq K(G, 1)$ be an aspherical, closed, smooth manifold with fundamental group $G$. The universal cover $\widetilde{B} \rightarrow B$ can serve as a model for the universal principal $G$-bundle $E G \rightarrow B G$ because it is a principal $G$-bundle with contractible total space $\widetilde{B}$, as follows from the fact that $\widetilde{B}$ is a simply connected CW-complex and $\pi_{i}(\widetilde{B}) \cong \pi_{i}(B)=0$ for $i \geq 2$. We recall that the Borel construction $F \times_{G} E G$ is the orbit space $(F \times E G) / G$, where 
$G$ acts diagonally on the product $F \times E G$. The $G$-equivariant cohomology of $F$ is the cohomology of the Borel construction,

$$
H_{G}^{\bullet}(F)=H^{\bullet}\left(F \times_{G} E G\right) .
$$

Using the model $\widetilde{B} \rightarrow B$, this may be computed as

$$
H_{G}^{\bullet}(F)=H^{\bullet}\left(F \times_{G} \widetilde{B}\right) .
$$

The space $F \times_{G} \widetilde{B}$ is the total space of a flat fiber bundle

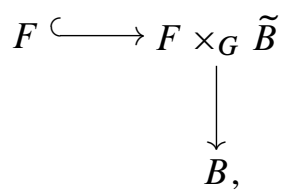

whose projection is induced by the second-factor projection $F \times \widetilde{B} \rightarrow \widetilde{B}$. Since $G$ acts isometrically on $F$, Theorem 5.2 applies and we conclude that the LerayCartan-Lyndon spectral sequence for real coefficients of the $G$-space $F$ collapses at the $E_{2}$-term. In particular,

$$
\begin{aligned}
H_{G}^{k}(F ; \mathbb{R}) & \cong H^{k}\left(F \times_{G} \widetilde{B} ; \mathbb{R}\right) \\
& \cong \bigoplus_{p+q=k} H^{p}\left(B ; \boldsymbol{H}^{q}(F ; \mathbb{R})\right) \\
& \cong \bigoplus_{p+q=k} H^{p}\left(G ; \boldsymbol{H}^{q}(F ; \mathbb{R})\right) .
\end{aligned}
$$

Remark 7.2. If $F \stackrel{i}{\rightarrow} E \rightarrow B$ is any fibration with $B$ path-connected such that the restriction $i^{*}: H^{\bullet}(E ; \mathbb{R}) \rightarrow H^{\bullet}(F ; \mathbb{R})$ is surjective (i.e. the fiber is "totally nonhomologous to zero in $E^{\prime \prime}$ ), then the action of $\pi_{1} B$ on $H^{\bullet}(F ; \mathbb{R})$ is trivial (as the map $f_{\gamma}: F_{b} \rightarrow F_{b^{\prime}}$ associated to a path $\gamma$ in $B$ from $b$ to $b^{\prime}$ comes, by the homotopy lifting property, with a homotopy $F_{b} \times I \rightarrow E$ between the inclusion of $F_{b}$ in $E$ and $f_{\gamma}$ followed by the inclusion of $F_{b^{\prime}}$ in $E$ ) and the spectral sequence of the fibration collapses at $E_{2}$, yielding the Leray-Hirsch theorem ([11], Thm. 5.10, p. 148). Our results apply to situations where $i^{*}$ needs not be surjective. In Section 8, we consider an example of a certain $\mathbb{Z}^{3}$-action on the manifold $F^{4}=S^{2} \times S^{2}$. This action is nontrivial even on cohomology and thus $i^{*}$ is not surjective in this example. Indeed, if $\left[S^{2}\right] \in H_{2}\left(S^{2}\right)$ is the fundamental class and $u \in H^{2}\left(S^{2} ; \mathbb{R}\right)$ denotes the generator with $\left\langle u,\left[S^{2}\right]\right\rangle=1$, then for instance $u \times 1 \in H^{2}\left(F^{4} ; \mathbb{R}\right)$ is not in the image of $i^{*}$.

Let us discuss some immediate consequences of Theorem 7.1. For a $G$-module $H$, let $H^{G}=\{v \in H \mid g v=v$ for all $g \in G\}$ denote the subspace of invariant elements. 
Corollary 7.3. Let $F$ be an oriented, closed, connected, smooth manifold and $G$ a discrete group as in Theorem 7.1, acting smoothly on F so that hypothesis (1) or (2) is satisfied. Then there is a monomorphism

$$
H^{k}(G ; \mathbb{R}) \oplus H^{k}(F ; \mathbb{R})^{G} \hookrightarrow H_{G}^{k}(F ; \mathbb{R})
$$

for $k \geq 1$.

Proof. For $k \geq 1$, the direct sum of Theorem 7.1 contains the term $H^{k}\left(G ; \boldsymbol{H}^{0}(F ; \mathbb{R})\right)$ and the term $H^{0}\left(G ; \boldsymbol{H}^{k}(F ; \mathbb{R})\right)$. The former is isomorphic to $H^{k}(G ; \mathbb{R})$, since $G$ acts trivially on $H^{0}(F ; \mathbb{R})$ and $H^{0}(F ; \mathbb{R}) \cong \mathbb{R}$ as $F$ is connected. The latter is isomorphic to $H^{k}(F ; \mathbb{R})^{G}$.

In particular, we obtain lower bounds for the ranks of the equivariant groups in terms of the ranks of the group cohomology.

Corollary 7.4. In the situation of Corollary 7.3, the inequalities

$$
\text { rk } H^{k}(G) \leq \operatorname{rk} H_{G}^{k}(F)
$$

hold for $k \geq 0$.

If a $G$-space $F$ has a fixed point, then $\pi: F \times{ }_{G} E G \rightarrow B G$ has a section given by $[e] \mapsto[(f, e)]$, where $f$ is a fixed point. Consequently, $\pi^{*}: H^{\bullet}(G) \rightarrow H_{G}^{\bullet}(F)$ is a monomorphism. Note that our results concern group actions that may be fixed-pointfree, even free. (See Example 7.6 below.) Let $\operatorname{cd}_{\mathbb{R}} G$ denote the $\mathbb{R}$-cohomological dimension of a group $G$, that is, the smallest $n \in \mathbb{N} \cup\{\infty\}$ such that $H^{k}(G ; \mathbb{R})$ vanishes for all $k>n$. For a topological space $X$, let $\operatorname{cd}_{\mathbb{R}} X$ be the smallest $n \in$ $\mathbb{N} \cup\{\infty\}$ such that the singular cohomology $H^{k}(X ; \mathbb{R})$ vanishes for all $k>n$.

Corollary 7.5. In the situation of Corollary 7.3, the inequality

$$
\operatorname{cd}_{\mathbb{R}} G \leq \operatorname{cd}_{\mathbb{R}}\left(F \times_{G} E G\right)
$$

holds.

Proof. Suppose that $n=\operatorname{cd}_{\mathbb{R}} G$ is finite. Then $H^{n}(G ; \mathbb{R})$ is not zero. By Corollary 7.4, $H_{G}^{n}(F ; \mathbb{R})$ is not zero and it follows that $\operatorname{cd}_{\mathbb{R}}\left(F \times_{G} E G\right) \geq n$. If $\operatorname{cd}_{\mathbb{R}} G=\infty$, then for every $n \in \mathbb{N}$, there exists an $N \geq n$ such that $H^{N}\left(F \times{ }_{G} E G ; \mathbb{R}\right) \neq 0$, whence $\operatorname{cd}_{\mathbb{R}}\left(F \times_{G} E G\right)=\infty$.

Although the underlying spaces of the $G$-actions considered in this paper are smooth manifolds, and hence can be given a (regular) $\mathrm{CW}$-structure, no such structure can usually be found that would make the $G$-space into a $G$-complex. A $G$-complex is a CW-complex together with a $G$-action which permutes the cells. A compact $G$-complex can be free only when $G$ is finite. But nontrivial finite groups cannot arise as fundamental groups of closed aspherical manifolds. On the other hand, free actions can occur in the context of Theorem 7.1, as the following example shows. 
Example 7.6. Let $G=\mathbb{Z}$ act freely on $F=S^{1}$ by powers of a rotation by an angle which is an irrational multiple of $2 \pi$. The quotient topology on the orbit space $S^{1} / \mathbb{Z}$ is the coarse topology, that is, the only open sets in $S^{1} / \mathbb{Z}$ are the empty set and $S^{1} / \mathbb{Z}$. The coarse topology on a set $X$ has the property that any map $Y \rightarrow X$ is continuous. In particular, the map $H: X \times I \rightarrow X$ given by $H(x, t)=x$ for $t \in[0,1)$ and $H(x, 1)=x_{0}$ for all $x \in X$, where $x_{0} \in X$ is a base-point, is continuous. Thus $X$ is homotopy equivalent to a point and therefore acyclic. This shows that $\operatorname{cd}_{\mathbb{R}}\left(S^{1} / \mathbb{Z}\right)=0$. Since $\operatorname{cd}_{\mathbb{R}} \mathbb{Z}=\operatorname{cd}_{\mathbb{R}} S^{1}=1$, the inequality $\operatorname{cd}_{\mathbb{R}} G \leq \operatorname{cd}_{\mathbb{R}}(F / G)$ does not hold and consequently, by Corollary 7.5, we have

$$
\operatorname{cd}_{\mathbb{R}}\left(F \times_{G} E G\right) \neq \operatorname{cd}_{\mathbb{R}}(F / G),
$$

despite the fact that the action is free. This also emphasizes that it is prudent to observe carefully the hypotheses of the Vietoris-Begle mapping theorem in attempting to apply it to the map $F \times_{G} E G \rightarrow F / G$ for a free action.

Example 7.6 illustrates once again that the actions considered in the present paper are generally not proper, since their orbits need not be closed. Furthermore, the isotropy groups for a proper $G$-CW complex are compact (so finite if $G$ is discrete). The isotropy groups arising for our actions can be infinite. (Consider the trivial $\mathbb{Z}$ action, or a $\mathbb{Z}$-action that factors through a finite cyclic group.) For a proper $G$-CW complex $X$, a result of W. Lück [9], Lemma 6.4, based on [10], Lemma 8.1, asserts that the projection $X \times_{G} E G \rightarrow X / G$ induces an isomorphism

$$
H_{n}\left(X \times_{G} E G ; \mathbb{Q}\right) \stackrel{\cong}{\rightrightarrows} H_{n}(X / G ; \mathbb{Q}) .
$$

For the actions arising in our Theorem 7.1, the groups $H_{n}\left(X \times_{G} E G ; \mathbb{Q}\right)$ and $H_{n}(X / G ; \mathbb{Q})$ are generally not isomorphic, as Example 7.6 shows.

Let us consider some specific groups.

Corollary 7.7. If $\mathbb{Z}^{n}$ acts isometrically on an oriented, closed, connected, Riemannian manifold $F$, then

$$
\operatorname{rk} H_{\mathbb{Z}^{n}}^{k}(F) \geq\left(\begin{array}{l}
n \\
k
\end{array}\right),
$$

with equality for $k=0$.

Proof. The inequality follows from Corollary 7.4 by observing that we may take $K\left(\mathbb{Z}^{n}, 1\right)=T^{n}$, the $n$-torus, and rk $H^{k}\left(T^{n}\right)=\left(\begin{array}{l}n \\ k\end{array}\right)$.

Corollary 7.8. If a discrete, integral Heisenberg group $\mathfrak{H}_{n}, n$ a positive integer, acts isometrically on an oriented, closed, connected, Riemannian manifold $F$, then rk $H_{\mathfrak{S}_{n}}^{0}(F)=1$,

$$
\text { rk } H_{\mathfrak{S}_{n}}^{k}(F) \geq 2 \text { for } k=1,2,
$$

and $H_{\mathfrak{S}_{n}}^{3}(F ; \mathbb{R})$ does not vanish. 
Proof. Let $\mathfrak{H}(\mathbb{R}) \subset G L_{3}(\mathbb{R})$ be the continuous Heisenberg group, i.e. the subgroup of upper triangular matrices of the form

$$
\left(\begin{array}{ccc}
1 & x & z \\
0 & 1 & y \\
0 & 0 & 1
\end{array}\right), \quad x, y, z \in \mathbb{R}
$$

This is a contractible Lie group. The discrete Heisenberg group $\mathfrak{S}_{n}$ can be described as the subgroup of $\mathfrak{H}(\mathbb{R})$ generated by the matrices

$$
X=\left(\begin{array}{ccc}
1 & 1 & 0 \\
0 & 1 & 0 \\
0 & 0 & 1
\end{array}\right), \quad Y_{n}=\left(\begin{array}{ccc}
1 & 0 & 0 \\
0 & 1 & n \\
0 & 0 & 1
\end{array}\right), \quad Z=\left(\begin{array}{lll}
1 & 0 & 1 \\
0 & 1 & 0 \\
0 & 0 & 1
\end{array}\right)
$$

It is a torsion-free, nilpotent group and a central extension of $\mathbb{Z}^{2}$ by $\mathbb{Z}$ with relations $\left[X, Y_{n}\right]=Z^{n},[X, Z]=1,\left[Y_{n}, Z\right]=1$. Being a subgroup of $\mathfrak{H}(\mathbb{R}), \mathfrak{S}_{n}$ acts freely (and properly discontinuously and cocompactly) on $\mathfrak{H}(\mathbb{R})$. Thus the quotient map $\mathfrak{H}(\mathbb{R}) \rightarrow B$ is the universal cover of the orbit space $B=\mathfrak{H}(\mathbb{R}) / \mathfrak{S}_{n}$ and $B$ is a closed, orientable, smooth 3-manifold, in fact, an orientable circle-bundle over the 2-torus. Moreover, $\pi_{1}(B)=\mathfrak{S}_{n}$ and $\pi_{k}(B)=\pi_{k} \mathfrak{H}(\mathbb{R})=0$ for $k \geq 2$. Hence $B=B \mathfrak{S}_{n}=K\left(\mathfrak{S}_{n}, 1\right)$ and we have

$$
\begin{aligned}
H_{1}\left(\mathfrak{S}_{n}\right) & =H_{1}(B)=\pi_{1} B /\left[\pi_{1} B, \pi_{1} B\right]=\mathfrak{H}_{n} /\left[\mathfrak{H}_{n}, \mathfrak{S}_{n}\right] \\
& =\left\langle X, Y_{n}, Z \mid\left[X, Y_{n}\right]=1,[X, Z]=1,\left[Y_{n}, Z\right]=1, Z^{n}=1\right\rangle \\
& =\mathbb{Z}^{2} \oplus \mathbb{Z} / n .
\end{aligned}
$$

(See also [1], Chap. I, Sec. 3 .) Thus

$$
\text { rk } H^{1}\left(\mathfrak{S}_{n}\right)=\operatorname{rk} H_{1}\left(\mathfrak{S}_{n}\right)=2 .
$$

By Poincaré duality, rk $H^{2}\left(\mathfrak{S}_{n}\right)=\operatorname{rk} H^{1}\left(\mathfrak{S}_{n}\right)$. Furthermore, rk $H^{3}\left(\mathfrak{S}_{n}\right)=1$, as $B$ is connected, closed, and orientable. The result follows from Corollary 7.4.

\section{An example}

We will compute the equivariant second real cohomology of a certain isometric $\mathbb{Z}^{3}$ action on $F^{4}=S^{2} \times S^{2}$, using our Theorem 7.1. The Eilenberg-MacLane space $K\left(\mathbb{Z}^{3}, 1\right)$ may be taken to be a 3-torus $T^{3}$. Viewing $S^{2}$ as the unit sphere in $\mathbb{R}^{3}$, the rotation group $\mathrm{SO}(3)$ acts linearly and isometrically on $S^{2}$. The metric on $F^{4}$ is the product metric. Let $R_{1}, R_{2}, R_{3} \in \mathrm{SO}(3)$ be three mutually commuting rotations, such as for example

$$
R_{i}=\left(\begin{array}{ccc}
\cos \phi_{i} & -\sin \phi_{i} & 0 \\
\sin \phi_{i} & \cos \phi_{i} & 0 \\
0 & 0 & 1
\end{array}\right)
$$


where the rotation angles $\phi_{i}, i=1,2,3$, are irrational multiples of $2 \pi$. We will specify the action of $G=\mathbb{Z}^{3}$ on $F^{4}$ by declaring how the generators $a=(1,0,0)$, $b=(0,1,0), c=(0,0,1) \in \mathbb{Z}^{3}$ act. If $(x, y)$ is a point in $S^{2} \times S^{2}, x, y \in S^{2}$, then set

$$
a(x, y)=\left(R_{1} y, x\right), \quad b(x, y)=\left(R_{2} x, R_{2} y\right), \quad c(x, y)=\left(R_{1} R_{3} y, R_{3} x\right) .
$$

It is readily verified that these three isometries of $F^{4}$ form a commuting set. The condition on the rotation angles implies that this action does not factor through a finite group. On $H^{0}\left(F^{4}\right)$, all elements of $G$ act trivially. Let $u \in H^{2}\left(S^{2}\right)$ be the generator with $\left\langle u,\left[S^{2}\right]\right\rangle=1$. Then $H^{2}\left(F^{4}\right)$ has rank 2 with basis $\{u \times 1,1 \times u\}$ and $H^{4}\left(F^{4}\right)$ has rank 1 generated by $u \times u$. The group operates on these classes by

$$
\begin{array}{lll}
a^{*}(u \times 1)=1 \times u, & b^{*}(u \times 1)=u \times 1, & c^{*}(u \times 1)=1 \times u, \\
a^{*}(1 \times u)=u \times 1, & b^{*}(1 \times u)=1 \times u, & c^{*}(1 \times u)=u \times 1 .
\end{array}
$$

The action on $H^{4}\left(F^{4}\right)$ is trivial; for example

$a^{*}(u \times u)=a^{*}((u \times 1) \cup(1 \times u))=a^{*}(u \times 1) \cup a^{*}(1 \times u)=(1 \times u) \cup(u \times 1)=u \times u$.

We calculate the twisted group $H^{0}\left(T^{3} ; \boldsymbol{H}^{2}\left(F^{4}\right)\right)$ :

$$
\begin{aligned}
H^{0}\left(T^{3} ; \boldsymbol{H}^{2}\left(F^{4}\right)\right)= & H^{2}\left(F^{4}\right)^{G} \\
= & \{\lambda(u \times 1)+\mu(1 \times u) \mid \lambda, \mu \in \mathbb{R}, g(\lambda(u \times 1)+\mu(1 \times u)) \\
& =\lambda(u \times 1)+\mu(1 \times u) \text { for all } g \in G\} \\
= & \{\lambda(u \times 1)+\mu(1 \times u) \mid \lambda(1 \times u)+\mu(u \times 1) \\
& =\lambda(u \times 1)+\mu(1 \times u)\} \\
= & \{\lambda(u \times 1)+\mu(1 \times u) \mid \lambda=\mu\} .
\end{aligned}
$$

Thus $H^{0}\left(T^{3} ; \boldsymbol{H}^{2}\left(F^{4}\right)\right)$ has rank 1 and is generated by $u \times 1+1 \times u$. Since $H^{1}\left(F^{4}\right)=$ 0 , we have $H^{1}\left(T^{3} ; \boldsymbol{H}^{1}\left(F^{4}\right)\right)=0$. Furthermore, as $\boldsymbol{H}^{0}\left(F^{4}\right)$ is a constant local system of rank one on $T^{3}$, the group $H^{2}\left(T^{3} ; \boldsymbol{H}^{0}\left(F^{4}\right)\right)=H^{2}\left(T^{3} ; \mathbb{R}\right)$ has rank 3 generated by the dual basis of the homology basis $S^{1} \times S^{1} \times \mathrm{pt}, S^{1} \times \mathrm{pt} \times S^{1}$, and pt $\times S^{1} \times S^{1}$. By Theorem 7.1,

$$
\begin{aligned}
H_{\mathbb{Z}^{3}}^{2}\left(F^{4}\right) & =H^{0}\left(T^{3} ; \boldsymbol{H}^{2}\left(F^{4}\right)\right) \oplus H^{1}\left(T^{3} ; \boldsymbol{H}^{1}\left(F^{4}\right)\right) \oplus H^{2}\left(T^{3} ; \boldsymbol{H}^{0}\left(F^{4}\right)\right) \\
& =H^{2}\left(F^{4}\right)^{\mathbb{Z}^{3}} \oplus 0 \oplus H^{2}\left(T^{3}\right) \\
& \cong \mathbb{R}^{4}
\end{aligned}
$$

\section{References}

[1] A. Adem and J. Milgram, Cohomology of finite groups. 2nd ed., Grundlehren Math. Wiss. 309, Springer-Verlag Berlin 2004. Zbl 1061.20044 MR 2035696 
[2] M. Banagl, Intersection spaces, spatial homology truncation, and string theory. Lecture Notes in Math. 1997, Springer-Verlag, Berlin 2010. Zbl 1219.55001 MR 2662593

[3] M. Banagl, Foliated stratified spaces and a de Rham complex describing intersection space cohomology. Preprint 2011. arXiv:1102.4781

[4] R. Bott and L. W. Tu, Differential forms in algebraic topology. Grad. Texts in Math. 82, Springer-Verlag, New York 1982. Zbl 0496.55001 MR 658304 (83i:57016)

[5] G. E. Bredon, Sheaf theory. 2nd ed., Grad. Texts in Math. 170, Springer-Verlag, New York 1997. Zbl 0874.55001 MR 1481706

[6] X. Dai, Adiabatic limits, nonmultiplicativity of signature, and Leray spectral sequence. J. Amer. Math. Soc. 4 (1991), 265-321. Zbl 0736.58039 MR 1088332

[7] M. W. Davis and J.-C. Hausmann, Aspherical manifolds without smooth or PL structure. In Algebraic topology (Arcata, CA, 1986), Lecture Notes in Math. 1370, Springer, Berlin 1989, 135-142. Zbl 0673.57020 MR 1000373

[8] R. Hartshorne, Algebraic geometry. Grad. Texts in Math. 52, Springer-Verlag, New York 1977. Zbl 0367.14001 MR 0463157

[9] W. Lück, Rational computations of the topological $K$-theory of classifying spaces of discrete groups. J. Reine Angew. Math. 611 (2007), 163-187. Zbl 1144.55007 MR 2361088

[10] W. Lück, H. Reich, and M. Varisco, Commuting homotopy limits and smash products. $K$-Theory 30 (2003), 137-165. Zbl 1053.55004 MR 2064237

[11] J. McCleary, A user's guide to spectral sequences. Cambridge Stud. Adv. Math. 58, 2nd ed., Cambridge University Press, Cambridge 2001. Zbl 0959.55001 MR 1793722

[12] J. Milnor, On the existence of a connection with curvature zero. Comment. Math. Helv. 32 (1958), 215-223. Zbl 0196.25101 MR 0095518

[13] J. W. Milnor and J. D. Stasheff, Characteristic classes. Ann. of Math. Stud. 76, Princeton University Press, Princeton, N.J., 1974. Zbl 0298.57008 MR 0440554

[14] J. Müller, A Hodge-type theorem for manifolds with fibered cusp metrics. Geom. Funct. Anal. 21 (2011), 443-482. Zbl 1223.58004 MR 2795514

[15] R. S. Palais, On the existence of slices for actions of non-compact Lie groups. Ann. of Math. (2) 73 (1961), 295-323. Zbl 0103.01802 MR 0126506

[16] J. Smillie, Flat manifolds with non-zero Euler characteristics. Comment. Math. Helv. 52 (1977), 453-455. Zbl 0357.53021 MR 0461521

Received June 3, 2011; revised December 5, 2011

M. Banagl, Mathematisches Institut, Universität Heidelberg, Im Neuenheimer Feld 288, 69120 Heidelberg, Germany

E-mail: banagl@mathi.uni-heidelberg.de 